\title{
ORIGINAL ARTICLE CYP4A in tumor-associated macrophages promotes pre-metastatic niche formation and metastasis
}

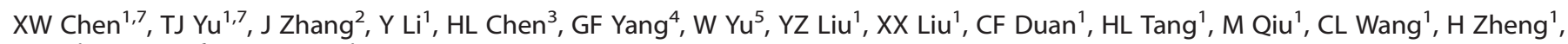
$\mathrm{J} \mathrm{Yue}^{1}, \mathrm{AM} \mathrm{Guo}^{6}$ and J Yang ${ }^{1}$

Tumor-associated macrophages (TAMs) play an essential role in metastasis. However, what enables TAMs to have a superior capacity to establish pre-metastatic microenvironment in distant organs is unclear. Here we have begun to uncover the effects of cytochrome P450 (CYP) 4A in TAMs on lung pre-metastatic niche formation and metastasis. CYP4A ${ }^{+}$TAM infiltration was positively associated with metastasis, pre-metastatic niche formation and poor prognosis in breast cancer patients. The pharmacological inhibition of CYP4A reduced lung pre-metastatic niche formation (evidenced by a decrease in vascular endothelial growth factor receptor 1 positive $\left(\mathrm{VEGFR} 1^{+}\right.$) myeloid cell recruitment and pro-metastatic protein expression) and metastatic burden, accompanied with TAM polarization away from the M2 phenotype in spontaneous metastasis models of 4T1 breast cancer and B16F10 melanoma. Co-implantation of 4T1 cells with CYP4A $10^{\text {high }}$ macrophages promoted lung pre-metastatic niche formation and metastasis. Depletion of TAMs disrupted lung pre-metastatic niches and thereby prevented metastasis. Treatment with the CM from CYP4A $10^{\text {high }}$ M2 macrophages (M2) increased pre-metastatic niche formation and metastatic burden in the lungs, whereas CYP4A inhibition attenuated these effects. In vitro TAM polarization away from the M2 phenotype induced by CYP4A inhibition decreased VEGFR $1^{+}$myeloid cell migration and fibronectin expression, accompanied with downregulation of STAT3 signaling. Conversely, overexpression of CYP4A or exogenous addition of 20-hydroxyeicosatetraenoic acid promoted M2 polarization and cytokine production of macrophages and thereby enhanced migration of VEGFR1 ${ }^{+}$myeloid cells, which were reversed by siRNA or pharmacological inhibition of STAT3. Importantly, a combined blocking M2 macrophage-derived factors TGF- $\beta$, VEGF and SDF-1 abolished VEGFR1 ${ }^{+}$myeloid cell migration and fibroblast activation induced by CYP4A. In summary, CYP4A in TAMs is crucial for lung pre-metastatic niche formation and metastasis, and may serve as a potential therapeutic target in human cancer.

Oncogene (2017) 36, 5045-5057; doi:10.1038/onc.2017.118; published online 8 May 2017

\section{INTRODUCTION}

Metastasis remains the leading cause of cancer-related deaths. ${ }^{1}$ Tumor metastasis needs to modify the local environment at future metastatic sites. Accordingly, a so-called pre-metastatic niche is induced by factors derived from the primary tumor before tumor cells arrive at the target organs. ${ }^{2}$ The recruitment of vascular endothelial growth factor receptor 1 (VEGFR1)-positive hematopoietic bone marrow progenitors, $C D 11 b^{+}$myeloid cells and suppressive immune cells, initiates the pre-metastatic niches and thereby promotes metastasis., ${ }^{3,4}$ Targeting the pre-metastatic niche-promoting molecular and cellular components to prevent metastasis may be an attractive approach for cancer therapeutics.

The tumor microenvironment is deeply engaged in determining the metastatic fate of the tumor. ${ }^{5}$ Tumor-associated macrophages (TAMs), one of the most abundant inflammatory stromal cells in the tumor microenvironment, ${ }^{6}$ exhibit predominantly M2-like protumor phenotype rather than M1-like anti-tumor phenotype, ${ }^{6}$ and their importance in metastasis is well established. ${ }^{7}$ Importantly, targeting TAMs prevents tumor metastasis through inhibiting production of various factors including chemokines, inflammatory and growth factors. ${ }^{8-10}$ Different types of immune cells, including myeloid-derived suppressor cells, regulatory $\mathrm{T}$ cells, and neutrophils, have been identified as contributing to pre-metastatic niche. ${ }^{3,11}$ Recruitment of monocytes/macrophages or stimulation of pulmonary alveolar macrophages by tumor-derived factors is essential for lung pre-metastatic niche establishment. ${ }^{12,13}$ However, the role of TAMs in forming a sanctuary for tumor cells in distant organs before tumor cell arrival has only begun to be appreciated.

20-Hydroxyeicosatetraenoic acid (20-HETE) is a novel lipid mediator contributing to tumor growth and angiogenesis. ${ }^{14}$ The 20-HETE-producing enzymes of cytochrome P450 (CYP) 4A families are upregulated in various human cancer tissues, including breast cancer, colon cancer and ovary cancer. ${ }^{15,16}$ Inhibition of 20-HETE synthesis by $N$-(4-butyl-2-methylphenyl)- $N^{\prime}$ hydroxyformamidine (HET0016) decreases pro-angiogenic factors and inhibits breast cancer growth. ${ }^{17}$ Our previous study showed that CYP4A11 overexpression in A549 human non-small-cell lung cancer cells enhances experimental lung metastasis. ${ }^{18}$ Downregulation of COX-2 and CYP4A signaling in human breast cancer cells inhibits metastasis through preventing anoikis resistance, migration and invasion. ${ }^{19}$ However, the effect of CYP4A/20-HETE

\footnotetext{
${ }^{1}$ Department of Pharmacology, School of Basic Medical Sciences, Wuhan University, Wuhan, China; ${ }^{2}$ Animal Experimental Center of Wuhan University, Wuhan, China; ${ }^{3}$ Department of Pathology and Pathophysiology, School of Basic Medical Sciences, Wuhan University, Wuhan, China; ${ }^{4}$ Department of Pathology, Zhongnan Hospital, Wuhan University, Wuhan, China; ${ }^{5}$ Department of Oncology, The First College of Clinical Medicine, China Three Gorges University, Yichang, China and ${ }^{6}$ Department of Pharmacology,

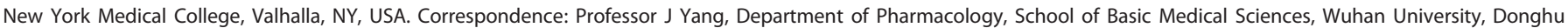
Road, Wuhan 430071, China.

E-mail: yangjingliu2013@163.com or jing_yang@whu.edu.cn

${ }^{7}$ These authors contributed equally to this work.

Received 11 August 2016; revised 7 March 2017; accepted 23 March 2017; published online 8 May 2017
} 
in not only TAMs but also likely other types of stromal cells on tumor metastasis and its mechanism has not been elucidated.

In this study, CYP4A expression in TAMs and its correlations with clinical outcome of patients with invasive breast carcinoma or melanoma were analyzed using human tumor tissue microarrays and The Cancer Genome Atlas (TCGA) cohort. The effects of CYP4A inhibition by HET0016 and dibromododecenyl methylsulfonimide (DDMS) (two relatively specific inhibitors of CYP4A) ${ }^{20}$ on premetastatic niche formation and metastasis were evaluated in spontaneous metastasis models of 4T1 breast cancer and B16F10 melanoma. In particular, we investigated whether CYP4A in TAMs contributes to pre-metastatic niche formation through promoting production of M2 macrophage-derived cytokines. Our results could lead to a novel strategy against tumor metastasis.

\section{RESULTS}

CYP4A protein is overexpressed in human breast cancer and melanoma

We first analyzed CYP4A protein expression in human breast cancer and melanoma using tissue microarrays, and found that CYP4A protein expression was higher in the invasive breast carcinoma than in the noninvasive breast carcinoma and benign tissues (Figures 1a and c). Consistently, CYP4A protein expression in the melanoma was elevated compared with the benign and normal skin tissues (Figures $1 \mathrm{~b}$ and $\mathrm{d}$ ). The correlations between CYP4A11/22 and clinical outcome of patients with invasive breast carcinoma $(n=780)$ or melanoma $(n=71)$ in TCGA cohort were explored. We found that high CYP4A22 expression was correlated with poor recurrence-free survival (RFS) but not overall survival (OS) in patients with invasive breast carcinoma (Figure 1e and Supplementary Figure 1a), while CYP4A11 expression had no significant correlation with OS and RFS (Supplementary Figures 1a and $b$ ). In the melanoma cohort, high expression of CYP4A11 was correlated with poor RFS (Figure 1f), but not correlated with OS (Supplementary Figure 1c). By using a Cox proportional hazards model, we examined the relationships between RFS and known prognostic factors, and found that CYP4A22 was a negative prognostic factor in human breast cancer (Supplementary Table 1). In the melanoma cohort, the patients with age $>64$ years, lymph node metastasis and tumor-node-metastasis
(TNM) stage III-IV did tend to have poor RFS, though the difference did not reach significance (Supplementary Table 2). Importantly, CYP4A11 was a valuable prognostic factor in human melanoma (Supplementary Table 2).

The correlations of CYP4A expression in TAMs with metastasis, pre-metastatic niche formation and poor prognosis

Similar to previous study, ${ }^{21} \mathrm{CD}^{+} 8^{+}$TAMs from human breast cancer and melanoma tissues mostly expressed M2 marker CD206, and accumulated at the tumor margin or within the peri-tumoral area (Supplementary Figure 1d). The co-expression of CYP4A with $\mathrm{CD}^{206^{+}}$macrophages showed that CYP4A protein mainly expressed in TAMs of M2 phenotype in tumor microenvironment (Supplementary Figure 1e). In addition, CYP4A11/22 mRNA expression and 20-HETE production were higher in breast TAMs (BCa TAMs) and melanoma TAMs (MEL TAMs) compared with monocyte-derived macrophages (MDMs) (Figures 2a and b). Consistently, robust expression of CYP4A11/22 and production of 20-HETE were observed in THP-1 macrophages induced by human breast cancer cells MDA-MB-231 (MDA-231-TAMs) or A375 human melanoma cells (A375-TAMs) compared with THP-1 macrophages (Figures $2 a$ and $b$ ).

To establish the pathological significance and clinical relevance of CYP4A expression in TAMs, a double immunofluorescent staining of CYP4A and CD206 was performed in a tissue microarray containing 140 human breast cancer tissues (Figure 2c). CYP4A ${ }^{+}$TAM infiltration was positively associated with higher tumor burden as defined by $\mathrm{T}$ stage as well as with aggressive tumor biology defined by pathological grade, lymph node metastasis and TNM stage (Table 1). Furthermore, reduced OS and RFS were observed in breast cancer patients with a high level of CYP4A ${ }^{+}$TAM infiltration in tumor tissues, which was also an independent prognostic parameter regarding RFS indicated by multivariate analysis (Figure $2 \mathrm{~d}$, Tables 2 and 3 ). The premetastatic niche plays a critical role in the metastatic process. ${ }^{2}$ Thus, we investigated the correlations of CYP4A ${ }^{+}$TAM infiltration with pre-metastatic niche markers in uninvolved (tumor cell-free) lymph nodes from 30 patients with invasive breast cancer. As shown in Figures $2 e$ and $f, C Y P 4 A^{+}$TAM infiltration in the primary tumor was significantly correlated with VEGFR $1^{+}$cell infiltration or typical pre-metastatic niche factor (S100A8 and fibronectin) levels a

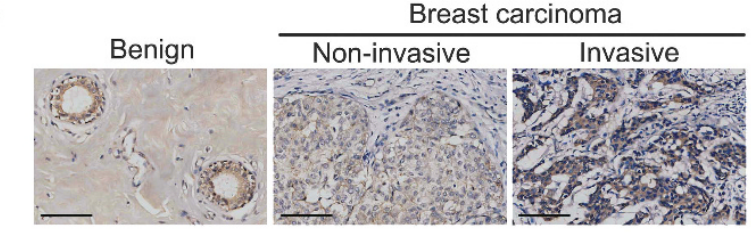

C

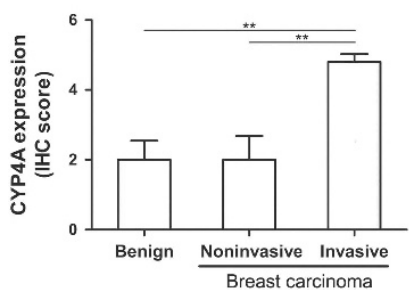

d

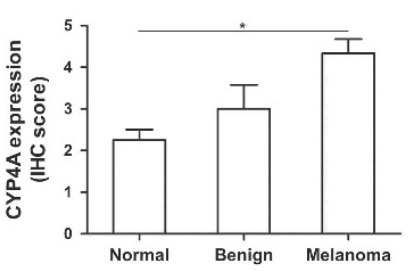

b

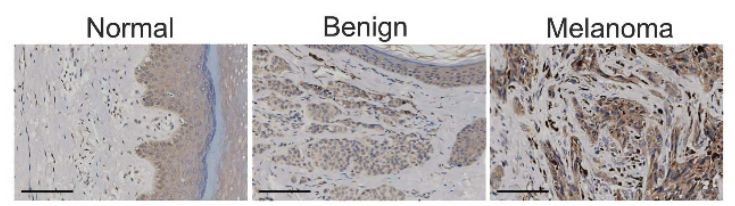

e

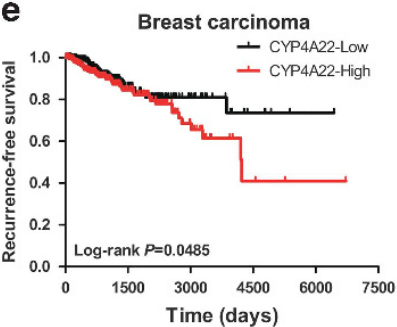

Figure 1. CYP4A protein is overexpressed in human breast cancer and melanoma. (a, b) Representative images of immunohistochemistry (IHC) staining of CYP4A protein in human breast cancer and melanoma tissue microarrays. Scale bar, $100 \mu \mathrm{m}$. (c) IHC scores of CYP4A staining in benign breast disease $(n=5)$, noninvasive breast carcinoma $(n=6)$ and invasive breast carcinoma tissues $(n=87)$. (d) IHC scores of CYP4A staining in normal skin $(n=4)$, benign $(n=4)$ and melanoma tissues $(n=27)$. ${ }^{*} P<0.05,{ }^{*} P<0.01$. (e) Kaplan-Meier recurrence-free survival (RFS) curves for breast carcinoma patients according to CYP4A22 expression in The Cancer Genome Atlas (TCGA) cohort ( $n=780)$. (f) KaplanMeier RFS curves for melanoma patients according to CYP4A11 expression in the TCGA cohort $(n=71)$. 

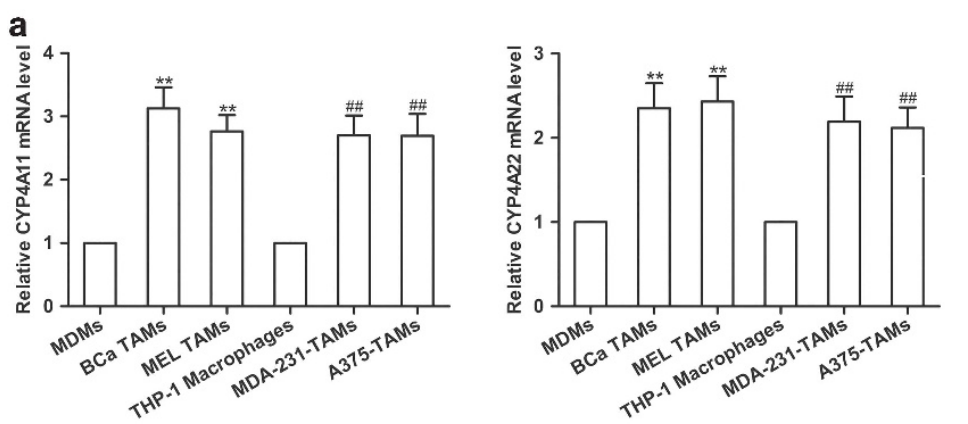
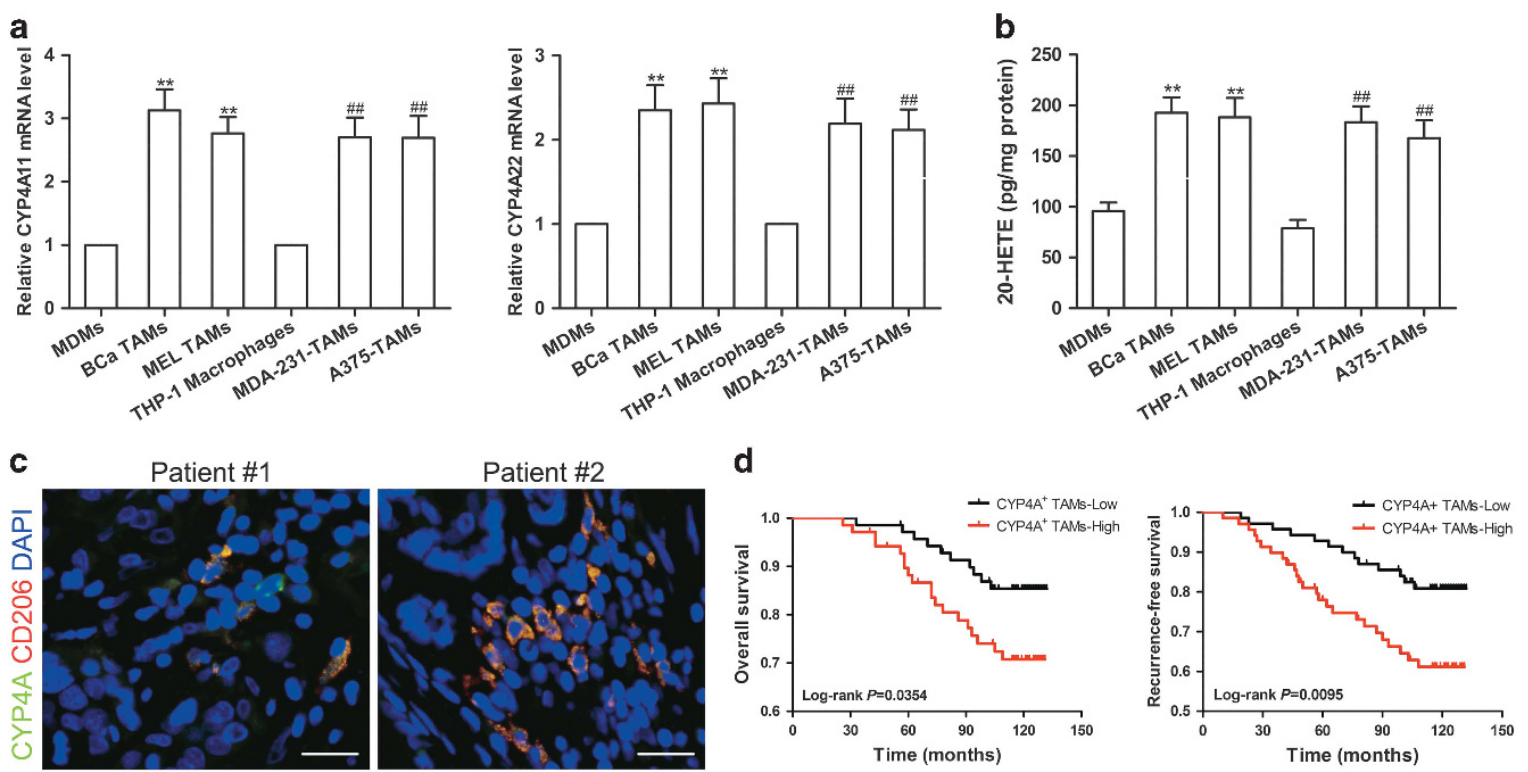

e

e Primary tumor Lymph node
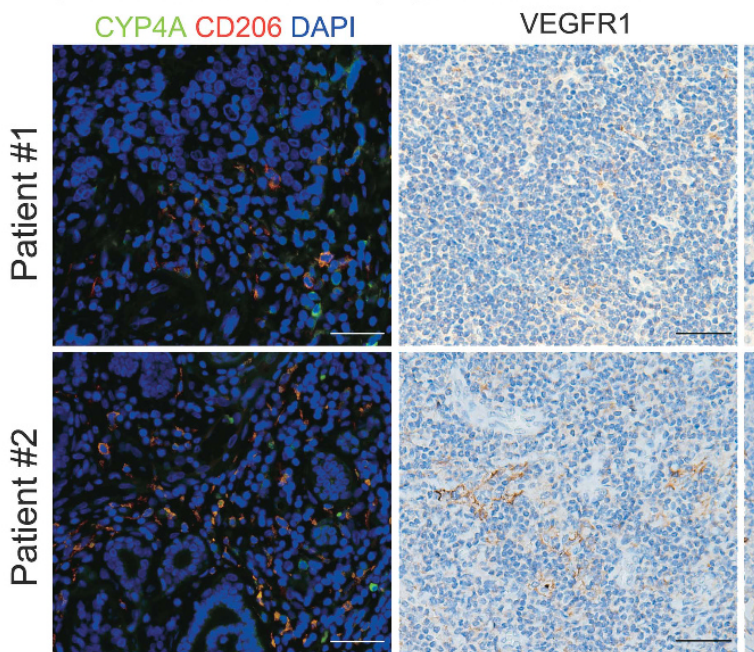

S100A8

Fibronectin
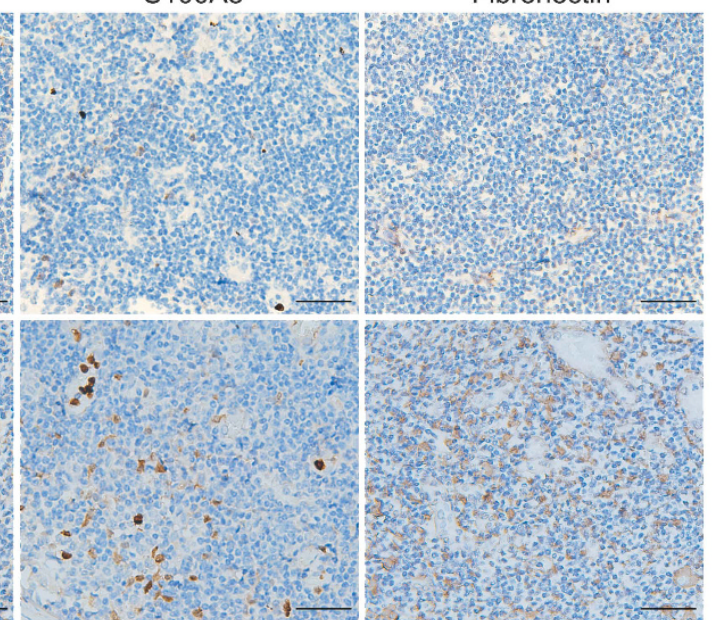

f
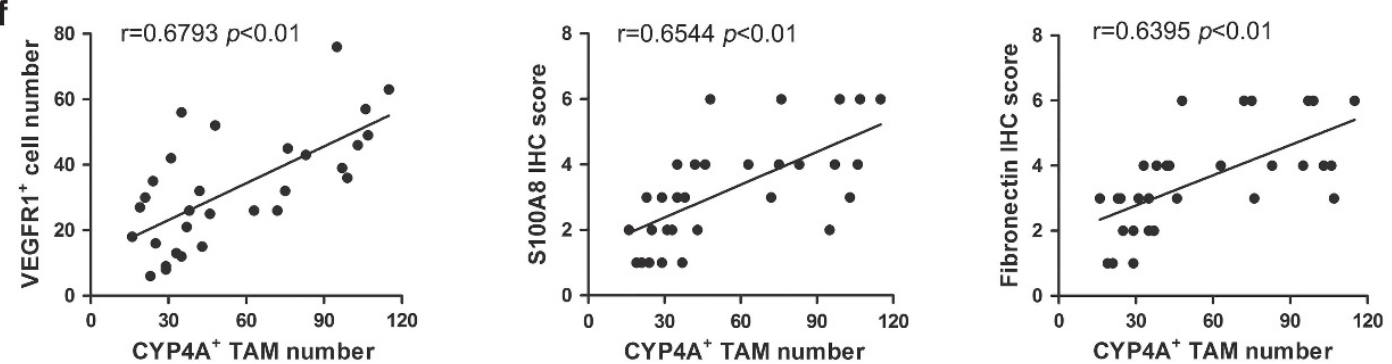

Figure 2. The correlations of CYP4A expression in TAMs with pre-metastatic niche markers and poor prognosis. (a) CYP4A11/22 mRNA expression in human monocyte-derived macrophages (MDMs), human breast TAMs (BCa TAMs), human melanoma TAMs (MEL TAMs), THP-1 macrophages, MDA-MB-231 human breast cancer cell-induced THP-1 macrophages (MDA-231-TAMs), or A375 human melanoma cell-induced THP-1 macrophages (A375-TAMs) $(n=8)$. (b) 20-HETE production in MDMs, BCa TAMs, MEL TAMs, THP-1 macrophages, MDA-231-TAMs and A375-TAMs was assayed by LC-ESI-MS/MS $(n=8)$. The value is presented as the mean \pm s.e.m. ${ }^{*} P<0.05,{ }^{* *} P<0.01$ vs $M D M s$, ${ }^{\#} P<0.05$, ${ }^{\# \#} P<0.01$ vs THP-1 macrophages. (c) Representative images of double immunofluorescence staining of CD206 (red) and CYP4A (green) in the human breast cancer tissue microarray. Scale bar, $20 \mu \mathrm{m}$. (d) Kaplan-Meier overall survival curves and recurrence-free survival curves for breast cancer patients according to the number of CYP4A ${ }^{+}$TAMs $(n=140)$. (e) Immunostaining of CYP4A, CD206, VEGFR1, S100A8 and fibronectin in

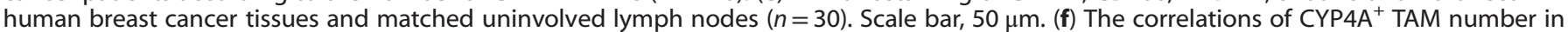
the primary tumor with VEGFR1, S100A8 and fibronectin levels in uninvolved lymph nodes from 30 patients with breast cancer. 
in uninvolved lymph nodes. These results together support the notion that CYP4A in M2-polarizated TAMs plays an essential role in pre-metastatic niche formation and metastasis in human cancer.

Table 1. The relationships between CYP4A ${ }^{+}$TAM counts and clinicopathological features in samples from 140 patients with breast cancer

\begin{tabular}{|c|c|c|c|c|c|}
\hline \multirow[t]{2}{*}{ Clinicopathological features } & \multirow[t]{2}{*}{ Cases } & \multicolumn{2}{|c|}{$\begin{array}{l}\text { CYP4A } A^{+} \text {TAM } \\
\text { counts }\end{array}$} & \multirow[t]{2}{*}{$\chi^{2}$} & \multirow[t]{2}{*}{$P$} \\
\hline & & Low & High & & \\
\hline \multicolumn{6}{|l|}{ Age (years) } \\
\hline$\leqslant 54$ & 71 & 35 & 36 & 0.029 & 0.866 \\
\hline$>54$ & 69 & 35 & 34 & & \\
\hline \multicolumn{6}{|l|}{ T stage } \\
\hline $\mathrm{T} 1$ & 57 & 36 & 21 & 6.658 & $0.010^{*}$ \\
\hline $\mathrm{T} 2+\mathrm{T} 3$ & 83 & 34 & 49 & & \\
\hline \multicolumn{6}{|l|}{ Lymph node metastasis } \\
\hline 0 & 75 & 45 & 30 & 6.462 & $0.011^{*}$ \\
\hline$\geqslant 1$ & 65 & 25 & 40 & & \\
\hline \multicolumn{6}{|l|}{ Pathological grade } \\
\hline |-II & 98 & 55 & 43 & 4.898 & $0.027^{*}$ \\
\hline III & 42 & 15 & 27 & & \\
\hline \multicolumn{6}{|l|}{ TNM stage } \\
\hline 1 & 33 & 25 & 8 & 11.459 & $0.001^{*}$ \\
\hline II-III & 107 & 45 & 62 & & \\
\hline \multicolumn{6}{|l|}{ ER status } \\
\hline Negative & 41 & 26 & 15 & 4.702 & $0.030^{*}$ \\
\hline Positive & 95 & 41 & 54 & & \\
\hline \multicolumn{6}{|l|}{ PR status } \\
\hline Negative & 64 & 41 & 23 & 11.043 & $0.001^{*}$ \\
\hline Positive & 73 & 26 & 47 & & \\
\hline \multicolumn{6}{|l|}{ Her-2 status } \\
\hline Negative & 61 & 34 & 27 & 3.784 & 0.052 \\
\hline Positive & 58 & 22 & 36 & & \\
\hline
\end{tabular}

CYP4A in TAMs promotes lung pre-metastatic niche formation and metastasis

A common route of tumor metastasis occurring is to the lung. Similar to previous study, ${ }^{13}$ the $4 \mathrm{~T} 1$-green fluorescent protein (GFP) cells and B16F10-GFP cells arrived to the mouse lungs, at the earliest, on days 18-20 after tumor implantation as a result of spontaneous metastasis of 4T1 breast cancer and B16F10 melanoma (data not shown). On day 28 after tumor implantation, CYP4A inhibition by HET0016 $(5.0 \mathrm{mg} / \mathrm{kg})$ and DDMS $(7.5 \mathrm{mg} / \mathrm{kg})$ decreased luciferase intensity, mCherry staining and metastatic nodules in the lungs, accompanied with obvious reduction in the tumor weight and 20-HETE production in TAMs (Figures 3a-e, Supplementary Figure 6a). On day 14 after tumor implantation, HET0016 $(5.0 \mathrm{mg} / \mathrm{kg})$ and DDMS $(7.5 \mathrm{mg} / \mathrm{kg})$ inhibited VEGFR1 ${ }^{+}$ myeloid cell cluster formation in the pre-metastatic lungs, accompanied with the decreased production of 20-HETE in TAMs (Figures $3 f$ and g). Circulating VEGFR $1^{+}$myeloid cells are potential clinical indicators of pre-metastatic niche formation. ${ }^{22,23}$ We found that VEGFR $1^{+}$myeloid cells were increased in the circulation on day 14 after tumor implantation, while CYP4A inhibition decreased these mobilizations (Supplementary Figure 2a). Several factors such as matrix metalloproteinase-9 (MMP-9), S100A8 and fibronectin mediate the pre-metastatic niche establishment. ${ }^{24-26} \mathrm{We}$ found that HET0016 and DDMS inhibited MMP-9, S100A8 and fibronectin expression in the pre-metastatic lungs without significantly influencing tumor growth (Figure $3 \mathrm{~h}$ and Supplementary Figure 6a), suggesting that lung pre-metastatic niche formation might be independent of the size of primary tumors. In another experiment, we analyzed the VEGFR1 $1^{+} \mathrm{GFP}^{+}$ myeloid cells in the lungs from the B16F10-bearing mice treated with or without HET0016 $(5.0 \mathrm{mg} / \mathrm{kg})$ or DDMS $(7.5 \mathrm{mg} / \mathrm{kg})$ by flow cytometry, and found that B16F10 melanoma promoted the recruitment of VEGFR $1^{+}$myeloid cells to the lungs, whereas, CYP4A inhibition by HET0016 and DDMS decreased this recruitment (Supplementary Figure $2 \mathrm{~b}$ ). These results suggest that the inhibition of CYP4A most likely in TAMs by HET0016 and DDMS reduces lung pre-metastatic niche formation, and consequently prevents metastasis.

To elucidate the direct effect of CYP4A in TAMs on premetastatic niche formation and metastasis, we injected 4T1 cells mixed with wild-type macrophages (control), negative control lentivirus macrophages (LV-NC) or CYP4A10 overexpressed (CYP4A10 $10^{\text {high }}$ ) macrophages into the right mammary fat pad of $\mathrm{BALB} / \mathrm{C}$ mice. As shown in Figures $4 \mathrm{a}-\mathrm{C}, \mathrm{CYP} 4 \mathrm{~A} 10^{\text {high }}$ in TAMs (Supplementary Figure 4d) increased tumor weight and lung metastatic nodules with an increase in 20-HETE production compared with the LV-NC group on day 28. On day 14, CYP4A $10^{\text {high }}$ significantly elevated the number of VEGFR $1^{+}$

Table 2. Univariate and multivariate Cox regression analysis of CYP4A+ TAM counts for overall survival of breast cancer patients in the tissue array

\begin{tabular}{|c|c|c|c|c|}
\hline \multirow[t]{2}{*}{ Characteristics } & \multicolumn{2}{|c|}{ Univariate analysis } & \multicolumn{2}{|c|}{ Multivariate analysis } \\
\hline & $H R(95 \% \mathrm{Cl})$ & P-value & $H R(95 \% \mathrm{Cl})$ & P-value \\
\hline Age $(\leqslant 54$ vs $>54)$ & $1.957(0.924-4.146)$ & 0.080 & & \\
\hline Pathological grade (I-II vs III) & $2.170(1.044-4.514)$ & $0.038^{*}$ & $1.097(0.420-2.865)$ & 0.850 \\
\hline T stage (T1 vs T2-T3) & $1.312(0.610-2.822)$ & 0.487 & & \\
\hline ER (negative vs positive) & $0.319(0.153-0.662)$ & $0.002^{*}$ & $0.361(0.150-0.868)$ & $0.023^{*}$ \\
\hline PR (negative vs positive) & $0.724(0.348-1.504)$ & 0.386 & & \\
\hline Her-2 (negative vs positive) & $0.381(0.159-0.912)$ & $0.030^{*}$ & $0.405(0.166-0.989)$ & $0.047^{*}$ \\
\hline Lymph node metastasis ( 0 vs $\geqslant 1$ ) & $2.789(1.270-6.128)$ & $0.011^{*}$ & $1.835(0.793-4.245)$ & 0.156 \\
\hline TNM stage (I vs II-III) & $2.954(0.894-9.764)$ & 0.076 & & \\
\hline CYP4A ${ }^{+}$TAMs counts (low vs high) & $2.227(1.035-4.791)$ & $0.041^{*}$ & $2.135(0.809-5.637)$ & 0.126 \\
\hline
\end{tabular}

Abbreviations: $\mathrm{Cl}$, confidence interval; ER, estrogen receptor; Her-2, human epidermal growth factor receptor-2; HR, hazard ratio; PR, progesterone receptor; TNM, tumor node metastasis. ${ }^{*} P<0.05$. 


\begin{tabular}{|c|c|c|c|c|}
\hline \multirow[t]{2}{*}{ Characteristics } & \multicolumn{2}{|c|}{ Univariate analysis } & \multicolumn{2}{|c|}{ Multivariate analysis } \\
\hline & $H R(95 \% \mathrm{Cl})$ & P-value & $H R(95 \% \mathrm{Cl})$ & P-value \\
\hline Age $(\leqslant 54$ vs $>54)$ & $1.107(0.586-2.092)$ & 0.753 & & \\
\hline Pathological grade (I-II vs III) & $2.278(1.201-4.322)$ & $0.012^{*}$ & $1.561(0.747-3.263)$ & 0.237 \\
\hline PR (negative vs positive) & $1.124(0.593-2.131)$ & 0.720 & & \\
\hline Her-2 (negative vs positive) & $0.955(0.483-1.891)$ & 0.895 & & \\
\hline Lymph node metastasis ( 0 vs $\geqslant 1$ ) & $2.963(1.494-5.879)$ & $0.002^{*}$ & $2.587(1.276-5.248)$ & $0.008^{*}$ \\
\hline TNM stage (I vs II-III) & $2.333(0.911-5.978)$ & 0.078 & & \\
\hline CYP4A $^{+}$TAM counts (low vs high) & $2.367(1.210-4.632)$ & $0.012^{*}$ & $2.221(1.041-4.735)$ & $0.039 *$ \\
\hline
\end{tabular}

myeloid cells and the levels of MMP-9, S100A8 and fibronectin in the pre-metastatic lungs with an increase in 20-HETE production (Figures 4c-e) without significantly affecting tumor growth (Figure 4b). Our findings suggest that CYP4A in TAMs contributes to pre-metastatic niche formation and metastasis.

CYP4A in TAMs is crucial for tumor-polarized macrophage phenotype switch

Given that TAM polarization plays a pivotal role in tumor metastasis, ${ }^{27}$ we first determined macrophage phenotypes in the murine models of 4T1 breast cancer and B16F10 melanoma. By double staining with anti-CYP4A antibody (red) and anti-CD206 antibody (green), we demonstrated a high level of CYP4A expression in TAMs of M2 phenotype in 4T1 and B16F10 tumor tissues (Supplementary Figure 3a), which is consistent with the data from human breast cancer and melanoma tissues. Intriguingly, significantly decreased $\mathrm{F} 4 / 80^{+} \mathrm{CD} 206^{+}$(M2) macrophage infiltration and elevated $\mathrm{F} 4 / 80^{+}{ }^{+} \mathrm{NOS}^{+}(\mathrm{M} 1)$ macrophage infiltration were observed in the HET0016- and DDMS-treated tumors compared to the model group (Figures $5 a$ and $b$, Supplementary Figures $3 \mathrm{~b}$ and $\mathrm{c}$ ), while there was no difference in $\mathrm{F} 4 / 80^{+}$macrophage infiltration among these groups (Figure $5 \mathrm{~b}$ ). We then measured representative M1 marker (iNOS) and M2 markers (CD206 and arginase-1) by quantitative real-time PCR (qPCR) and western blot, and found that CYP4A inhibition by HET0016 and DDMS decreased the levels of M2 markers, but increased the level of M1 marker in TAMs isolated from 4T1 and B16F10 tumor tissues (Figures 5c and d). Consistently, HET0016 and DDMS increased TNF- $a$ and IL-12 (Th1 cytokines) production, whereas, decreased TGF- $\beta$ and IL-10 (Th2 cytokines) production in TAMs (Figures $5 e$ and $f$ ). These data suggest that TAMs were predominantly of the M2-like, tumor-promoting phenotype, whereas CYP4A inhibition by HET0016 and DDMS skews TAMs away from the $\mathrm{M} 2$ phenotype to the $\mathrm{M} 1$ phenotype.

We found that CYP4A expression and 20-HETE production were increased during in vitro polarization of human MDMs, RAW264.7 cells and mouse peritoneal macrophages (PNMS) (MO macrophages) to the M2 macrophages (Figures $6 a$ and b). By contrast, HET0016 $(5 \mu \mathrm{M})$ and DDMS $(10 \mu \mathrm{M})$ downregulated the gene expression of M2 markers (CD206 and arginase-1) and inhibited secretion of Th2 cytokines (TGF- $\beta$ and IL-10), accompanied with a decrease in 20-HETE production (Figures $6 a-d$ ), without affecting the proliferation of MDMs, RAW264.7 cells and PNMS (Supplementary Figures $4 a-c$ ). Moreover, CYP4A overexpression enhanced M2 marker expression and Th2 cytokine production (Figures $6 \mathrm{c}$ and $d$ ), accompanied with the increased 20-HETE production (Supplementary Figure 4d). Consistently, immunofluorescence analysis showed that CD206 expression was significantly increased in CYP4A $10^{\text {high }}$ M2 macrophages, but decreased in HET0016- and DDMS-treated M2 macrophages (Figure 6e). These results suggest that CYP4A in TAMs is crucial for tumor-polarized macrophage phenotype switch.

CYP4A promotes TAM-induced pre-metastatic niche formation and metastasis

To clarify whether TAMs participate in pre-metastatic niche formation and metastasis, and such a role is related to their M1/ M2-like phenotype regulated by CYP4A, we treated 4T1 tumorbearing mice with Clodronate (Clod) and Zoledronic acid (ZA) liposomes to chemically deplete TAMs (using vehicle liposomes as control), and compared their effects in control tumors (containing more M2-polarizated TAMs) with HET0016-treated tumors (containing more M1-polarizated TAMs). Flow cytometry analysis of CD $11 \mathrm{~b}^{+}$cells in peripheral blood and immunohistochemical analysis of $\mathrm{F}_{4} / 80^{+}$macrophages in tumor tissues showed depletion of TAMs by Clod and ZA liposomes (Supplementary Figures $5 a-d)$. On day 14 after tumor implantation, depletion of TAMs significantly decreased the number of VEGFR $1^{+}$myeloid cells in peripheral blood and the levels of MMP-9, S100A8 and fibronectin in the pre-metastatic lungs (Supplementary Figures $5 \mathrm{e}$ and f). On day 28 after tumor implantation, depletion of TAMs significantly decreased the number of metastatic nodules in the lungs (Supplementary Figure $5 \mathrm{~g}$ ). However, TAM depletion failed to further affect the inhibition of lung pre-metastatic niche formation and metastasis by HET0016 (Supplementary Figure 5eg). These data indicate that TAMs may contribute to premetastatic niche formation and thereby promote metastasis, and M1-polarized TAMs in HET0016-treated tumors did not (prominently) regulate lung pre-metastatic niche formation and metastasis.

To support the results obtained in these in vivo depletion experiments, as depicted schematically, we used the conditioned medium (CM) from M2 macrophages (M2), HET0016 or DDMStreated M2, and CYP4A10 high M2 for further experiments (Figure 7a). As shown in Figures $7 b-d$, the $C M$ from $M 2$ or CYP4A $10^{\text {high }} \mathrm{M} 2$ increased the number of VEGFR $1^{+}$myeloid cells and the levels of pro-metastatic proteins in the lungs, whereas CYP4A inhibition by HET0016 and DDMS attenuated these effects. We then treated mice with the CM described above for 2 weeks following by $4 \mathrm{~T} 1$ cells challenge, and found that lung metastatic nodules were decreased in the HETO016- and DDMS-treated groups, but increased in the CYP4A $10^{\text {high }}$ group compared with the M2 CM-treated group (Figure 7e). Next, we determined whether TAMs could have a substantial impact on in vitro 

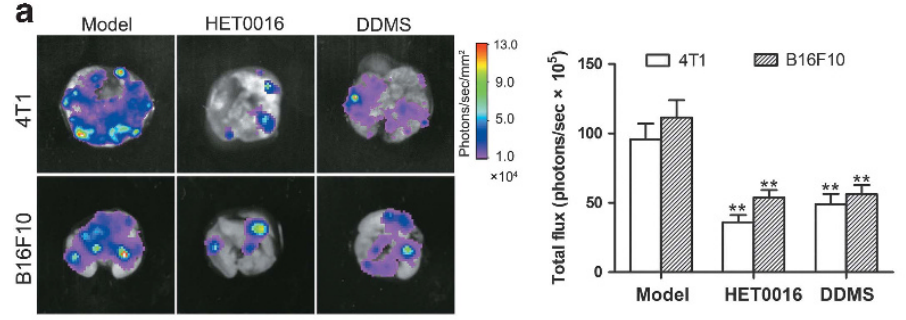

b

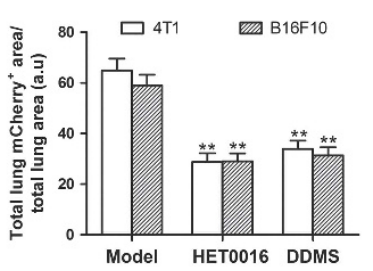

C

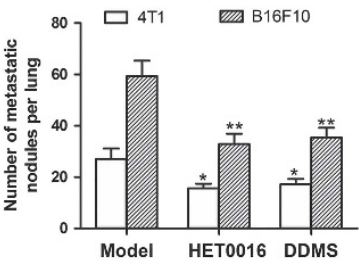

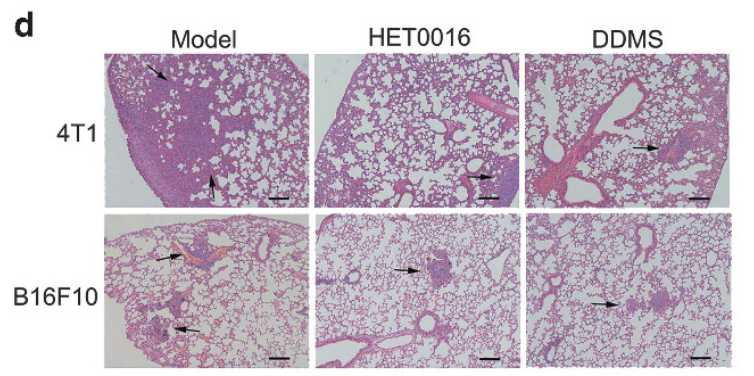

e

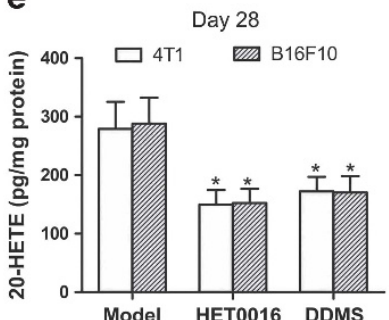

f

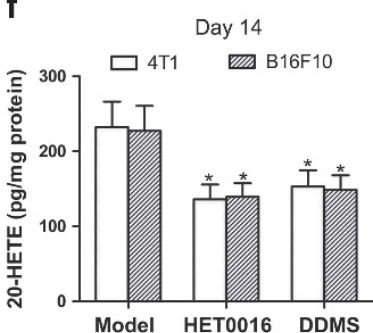

g
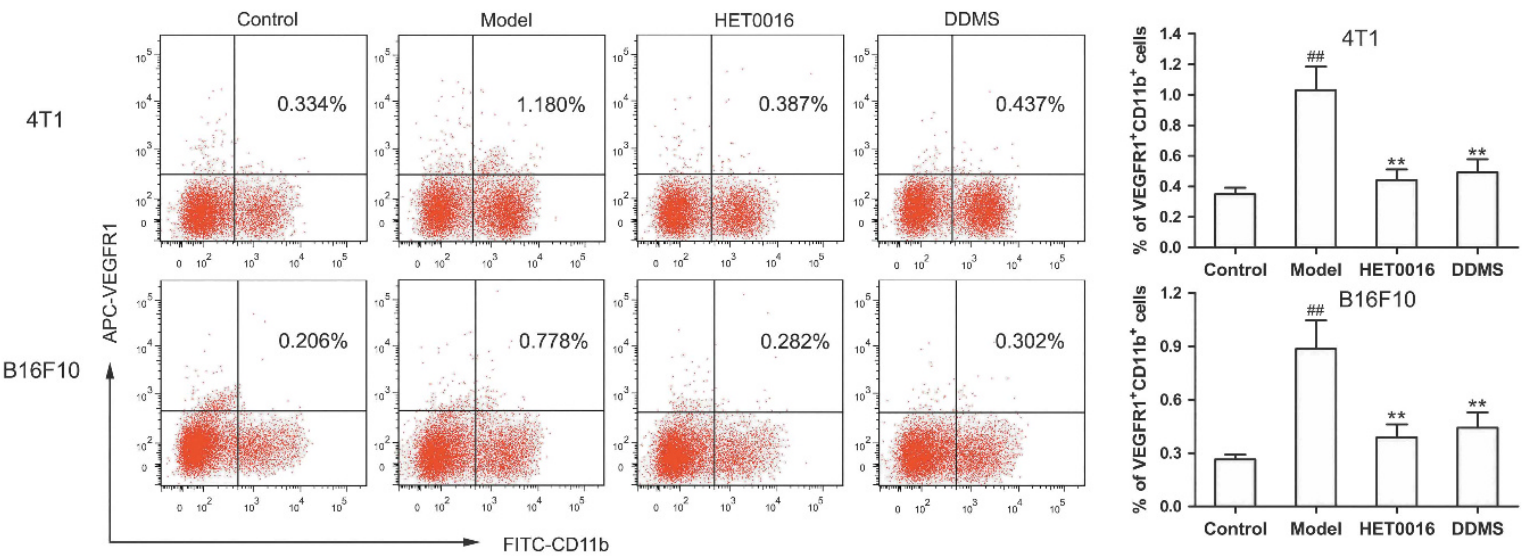

h

4T1
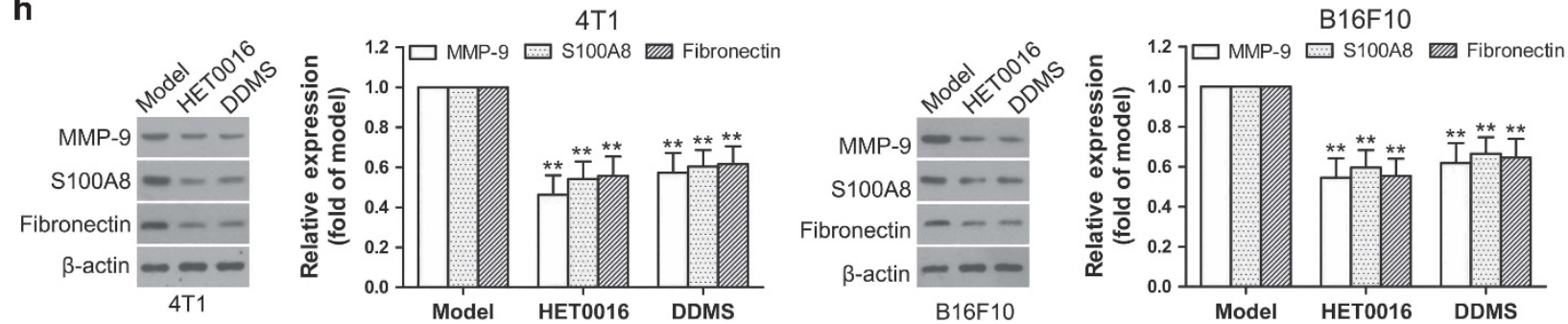

Figure 3. CYP4A inhibition suppresses lung pre-metastatic niche formation and metastasis in $4 \mathrm{~T} 1$ breast cancer and B16F10 melanoma. The $\mathrm{BALB} / \mathrm{c}$ mice or C57BL/6 mice were injected with 4T1-mCherry-luciferase cells or B16F10-mCherry-luciferase cells, respectively, and then treated with HET0016 $(5.0 \mathrm{mg} / \mathrm{kg})$, DDMS $(7.5 \mathrm{mg} / \mathrm{kg})$ or vehicle five times a week from the day after implantation. (a) On day 28 after tumor implantation, representative images and quantitative analysis of lung metastasis from the mice treated with or without HET0016 and DDMS detected by ex vivo luciferase-based bioluminescence imaging $(n=8)$. (b) mCherry immunohistochemistry (IHC) of lung sections from animals inoculated with the indicated cell lines. Lung $\mathrm{mCherry}^{+}$metastatic area was assayed by calculating the total area of lung $\mathrm{mCherry}^{+}$lesion normalized per the total area of the lungs $(n=8)$. Three sections were analyzed per lung. (c, d) Hematoxylin/eosin-stained lung sections and quantitative analysis of lung metastases $(n=8)$. Scale bars, $100 \mu \mathrm{m}$. (e) On day 28 after tumor implantation, 20-HETE production in tumorassociated macrophages (TAMs) from 4T1 and B16F10 tumor tissues was measured by LC-ESI-MS/MS ( $n=8$ ). (f) 20-HETE production in the TAMs was measured at 14 days after tumor inoculation $(n=8)$. (g) On day 14 after tumor implantation, VEGFR $1^{+}$myeloid cells in the lungs from tumor-free mice (control), tumor-bearing mice (model), and HET0016- and DDMS-treated mice were determined by flow cytometry $(n=5)$. (h) MMP-9, S100A8 and fibronectin expression in the lungs were determined by western blot $(n=8)$. The value is presented as the mean \pm s.e.m. ${ }^{\# \#} P<0.01$ vs control, ${ }^{*} P<0.05,{ }^{* *} P<0.01$ vs model.

fibroblast activation and myeloid cell migration, crucial steps for pre-metastatic niche formation. ${ }^{4}$ Figure $7 f$ showed that CYP4A inhibition by HET0016 and DDMS decreased the M2 CM-induced migration of VEGFR $1^{+}$myeloid cells. Furthermore, treatment of L929 mouse fibroblast and primary fibroblast derived from mouse lungs (LF) with the M2-CM induced a-smooth muscle actin (aSMA) (a marker of fibroblast activation) and fibronectin expression, whereas, CYP4A inhibition attenuated these effects (Figure $7 \mathrm{~g}$ ). These data suggest that CYP4A promotes TAM-induced premetastatic niche formation and metastasis. 

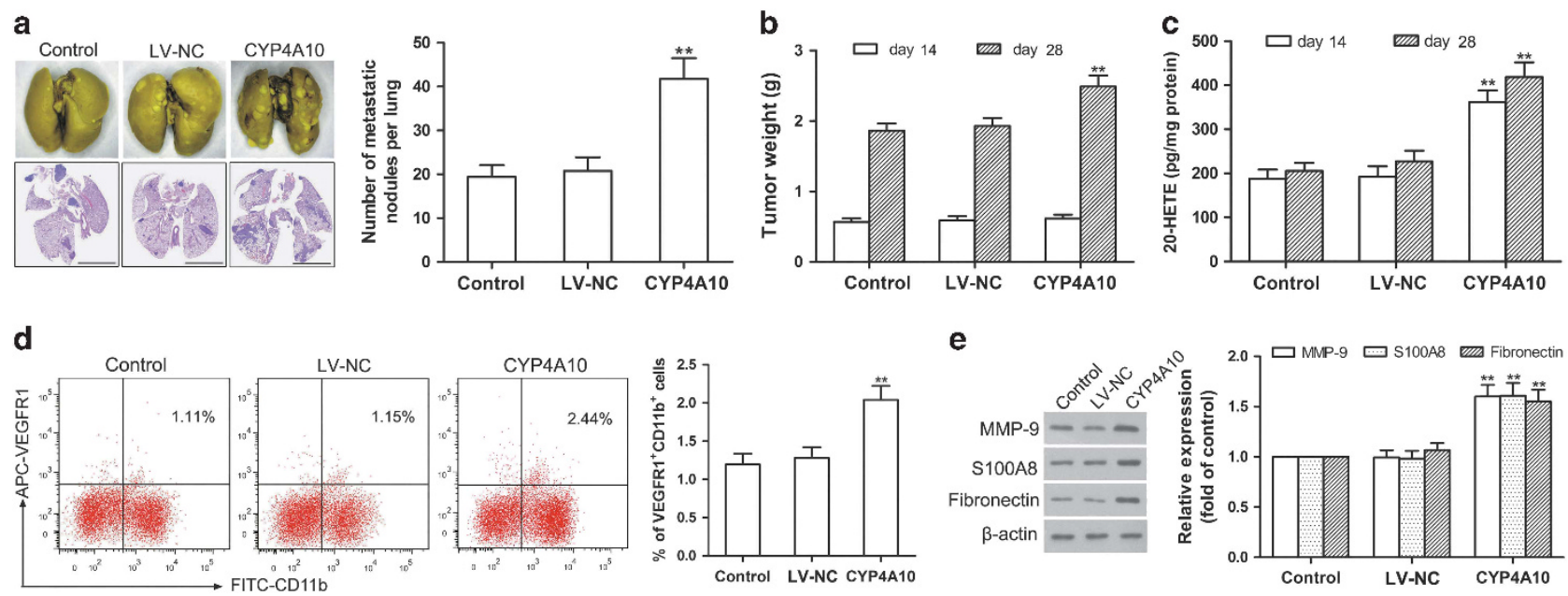

Figure 4. Co-implantation of $4 \mathrm{~T} 1$ cells with CYP4A $10^{\text {high }}$ macrophages promotes lung pre-metastatic niche formation and metastasis. $4 \mathrm{~T} 1$ cells $\left(6 \times 10^{5}\right)$ mixed with wild-type RAW264.7 cells, negative control lentivirus (LV-NC)-treated RAW264.7 cells or CYP4A10 high RAW264.7 cells $\left(2 \times 10^{5}\right)$ were injected into the right mammary fat pads of BALB/c mice in a ratio of 3:1. (a) Hematoxylin/eosin-stained lung sections and quantification of lung metastase on day 28 after tumor implantation $(n=8)$. Scale bars, $5 \mathrm{~mm}$. (b) The tumor weight was measured on day 14 and day 28 after tumor implantation $(n=8)$. (c) 20-HETE production in TAMs on day 14 or day 28 were determined by LC-ESI-MS/MS $(n=8)$. (d) VEGFR $1^{+}$myeloid cells in the pre-metastatic lungs were analyzed by flow cytometry on day $14(n=5)$. (e) MMP-9, S100A8 and fibronectin in the pre-metastatic lungs were determined by western blot $(n=8)$. The value is presented as the mean \pm s.e.m. ${ }^{*} P<0.05$, ${ }^{* *} P<0.01$ vs $\mathrm{LV}$-NC.

TAM-derived factors regulated by CYP4A drive lung pre-metastatic niche formation

TAMs secrete a wide variety of cytokines that are associated with tumor metastasis. ${ }^{5}$ Thus, we used qPCR to identify M2 macrophage-derived factors known to promote metastasis. As shown in Figure $8 \mathrm{a}, \mathrm{TGF}-\beta, \mathrm{VEGF}$, stromal cell-derived factor-1 (SDF-1), platelet-derived growth factor B (PDGF-B) and MMP-9 expression were increased in the CYP4A10 high $M 2$ compared with M2, but insulin-like growth factor 1 (IGF-1) and MMP-2 not. Tumor recruits bone marrow-derived cells through VEGF and SDF-1 secretion, thus promotes pre-metastatic niche formation. ${ }^{28}$ As expected, CYP4A10 over-expression increased VEGF and SDF-1 levels in M2 polarized macrophages (Figure 8b), whereas CYP4A inhibition by HET0016 and DDMS decreased levels of VEGF and SDF-1 in M2 polarized macrophages, 4T1 TAMs and B16F10 TAMs (Figure 8b, Supplementary Figures $6 \mathrm{c}$ and d). Importantly, neutralizing antibodies against VEGF and SDF-1 partly abolished CYP4A10-induced migration of VEGFR $1^{+}$myeloid cells (Figure 8c). TGF- $\beta$, VEGF, SDF-1, PDGF-B and MMP-9 activate fibroblasts to produce fibronectin. ${ }^{29-31}$ Consistent with this, CYP4A10-induced expression of a-SMA was partly abolished by neutralizing antibodies against TGF- $\beta$, VEGF, SDF-1, PDGF-B and MMP-9, especially TGF- $\beta$ (Figure $8 d$ ). Blockade of TGF- $\beta$ receptor also reversed CYP4A10-induced expression of $\alpha$-SMA and fibronectin (Figure 8e). A combined blocking of VEGF, SDF-1 and TGF- $\beta$ showed better efficacy than single blocking in abolishing fibroblast activation (Figure 8f). Interestingly, co-injection of the M2-CM with VEGF, SDF- 1 and TGF- $\beta$ reproduced similar recruitment of VEGFR $1^{+}$myeloid cells induced by the CM from CYP4A $10^{\text {high }}$ macrophages (Figure $8 \mathrm{~g}$ ). These results suggest that CYP4A in TAMs promotes pre-metastatic microenvironment formation through $\mathrm{M} 2$ macrophage-derived factors, especially including TGF- $\beta$, SDF-1 and VEGF.

CYP4A/20-HETE-STAT3 signaling in TAMs is critical for premetastatic niche formation

Signal transducers and activators of transcription 3 (STAT3) is involved in macrophage M2 polarization. ${ }^{32}$ We demonstrated the increased levels of p-STAT3 in MDMs- and PNMS-derived M2 macrophages (Figure 9a). In contrast, CYP4A inhibition by HET0016 and DDMS significantly decreased p-STAT3, accompanied with a decrease in CD206 expression (Figures 9b and c). Consistently, we observed the decreased p-STAT3 in TAMs isolated from the 4T1 and B16F10 tumor tissues treated with HET0016 and DDMS (Supplementary Figure 6b). Moreover, overexpression of CYP4A or exogenous addition of 20-HETE enhanced Th2 cytokine (TGF- $\beta$ and IL-10) production, which was reversed by Stattic (a STAT3 inhibitor) or STAT3-siRNA (Figures 9d-f). Importantly, treatment with stattic or STAT3-siRNA reversed VEGF and SDF- 1 production and VEGFR1 ${ }^{+}$myeloid cell migration induced by overexpression of CYP4A or exogenous addition of 20-HETE (Figures $9 \mathrm{~g}$ and $\mathrm{h}$ ). These results suggest that CYP4A/20HETE-STAT3 signaling is critical for macrophage M2 polarization and pre-metastatic niche formation.

\section{DISCUSSION}

Our previous study suggested that overexpression of CYP4A11 in human non-small-cell lung cancer cells promotes metastasis. ${ }^{18}$ However, isoliquiritigenin prevents metastasis through downregulation of COX-2 and CYP4A in human breast cancer cells. ${ }^{19}$ In the present study, two novel observations have been made. First, we have provided direct evidence that CYP4A in TAMs initiates pre-metastatic niche formation and consequently promotes metastasis in 4T1 breast cancer and B16F10 melanoma. To our knowledge, this is the first study that directly demonstrated that CYP4A in TAMs exerts the driving forces to 'fertilize' the distinct organ for metastasis through promoting production of $\mathrm{M} 2$ macrophage-derived cytokines (TGF- $\beta$, SDF- 1 and VEGF). Second, we have demonstrated for the first time that CYP4A induces the polarization of macrophages to M2 phenotype via STAT3 signaling. These results implicate CYP4A/20-HETE in TAMs as important mediators of disseminating tumor cells to colonize distant organs, highlighting the therapeutic potential of CYP4A/ 20-HETE in tumor microenvironment for interference of metastasis.

It was previously reported to prevent metastasis by eliminating pre-metastatic niches. ${ }^{33}$ Recent studies suggest a paradigmshifting concept that regulatory and suppressive immune cells are crucial in providing tumor cells a conducive microenvironment to engraft and colonize in distant organs. ${ }^{11,34,35}$ Here we demonstrated that CYP4A in TAMs correlated with metastasis, 
a

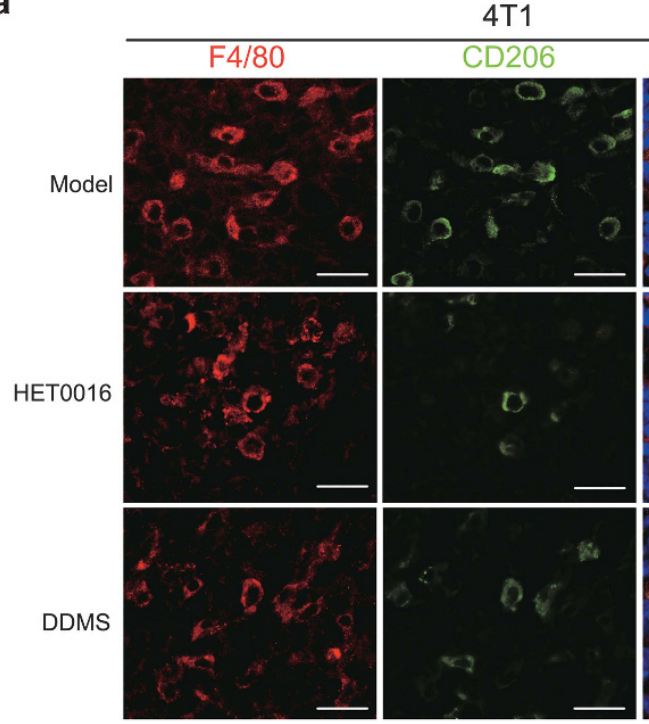

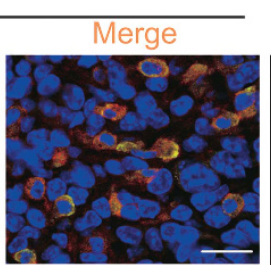
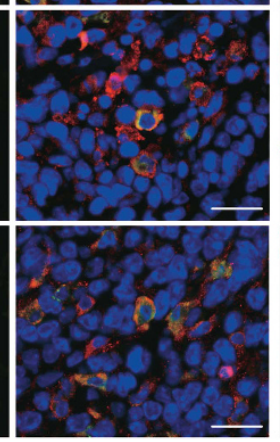

B16F10

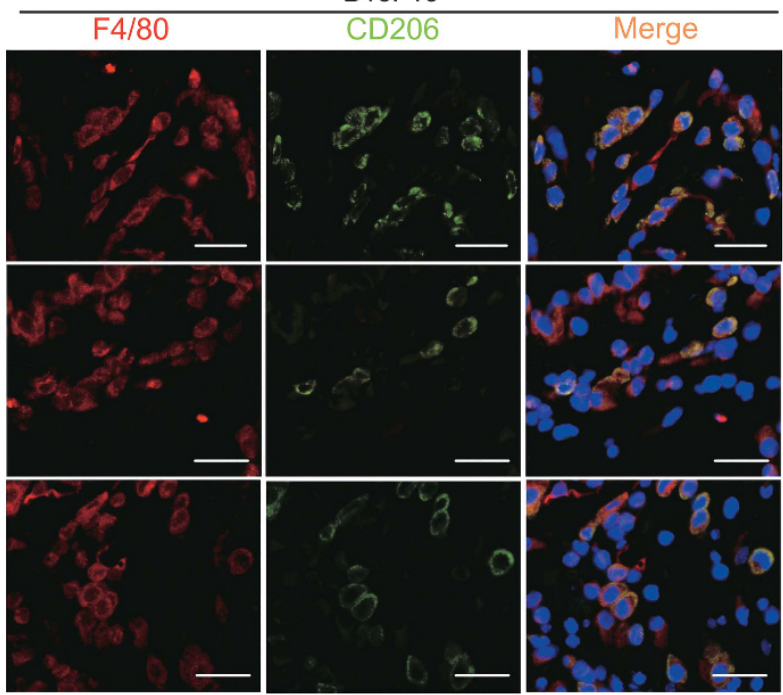

b

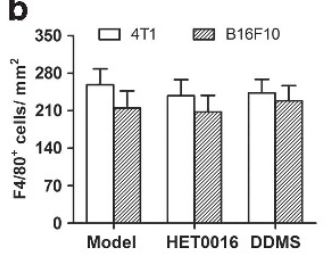

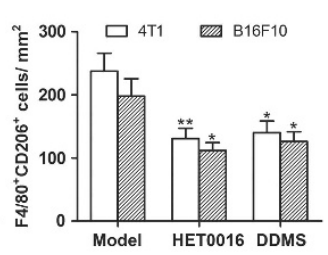
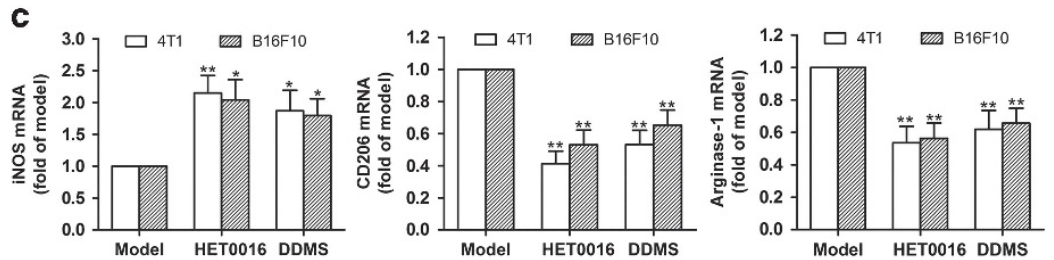

d
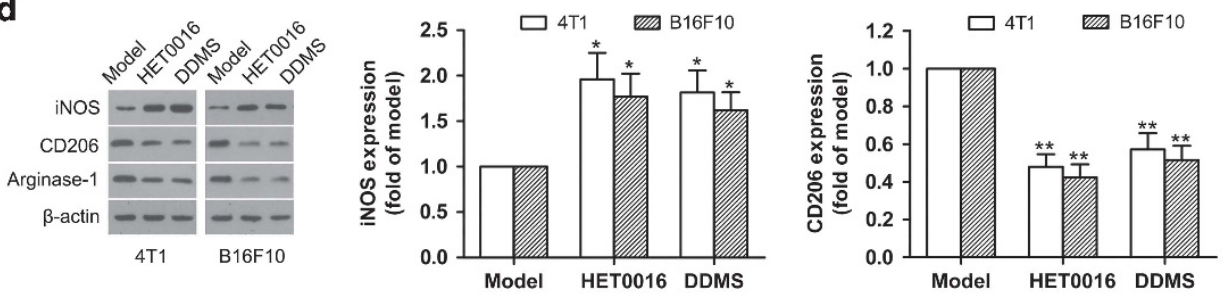

e
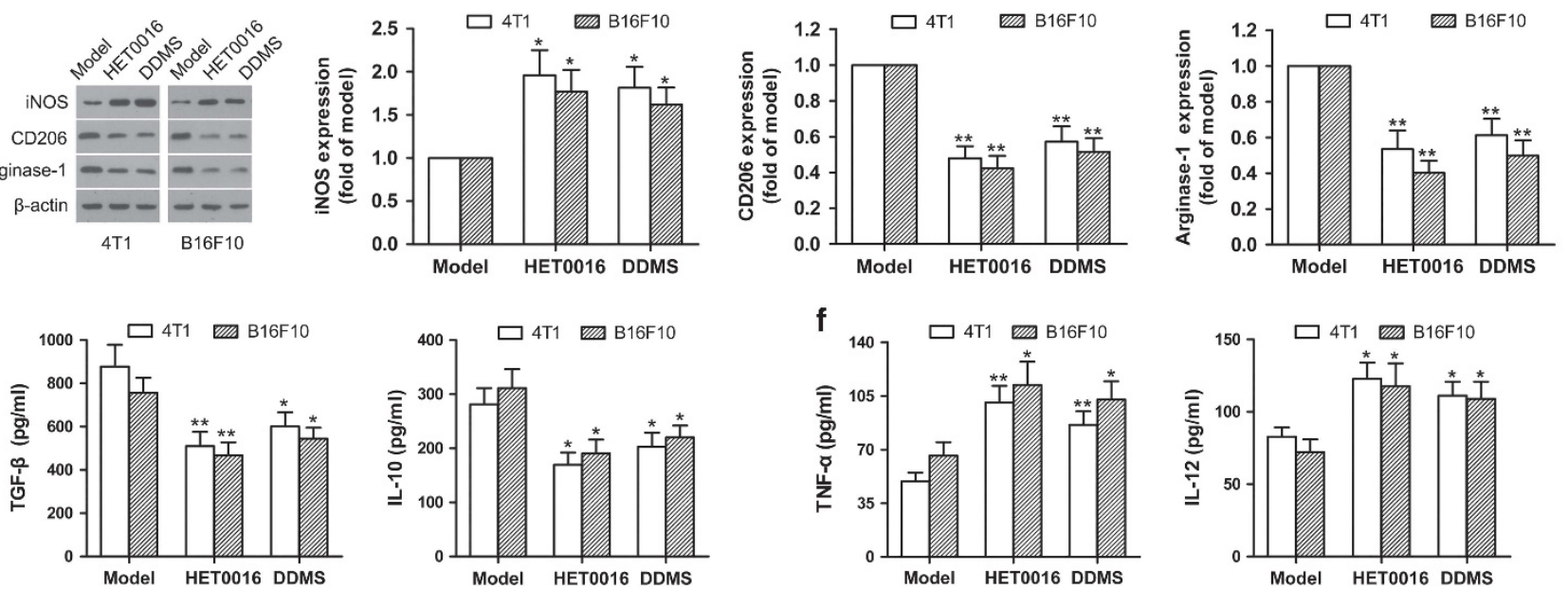

Figure 5. CYP4A inhibition regulates TAM polarization away from the M2 phenotype in 4T1 breast cancer and B16F10 melanoma. (a) Immunostaining analysis of $\mathrm{F} 4 / 80^{+} \mathrm{CD} 206^{+}$macrophages in the tumor tissues from the groups treated with or without HET0016 and DDMS was shown. Scale bars, $20 \mu \mathrm{m}$. (b) Corresponding quantitative analysis of tumor-infiltrating $\mathrm{F} 4 / 80^{+}$macrophages and $\mathrm{F} 4 / 80^{+} \mathrm{CD} 206^{+} \mathrm{M} 2$ macrophages $(n=8)$. (c) Relative mRNA levels of M1 marker (iNOS) and M2 markers (CD206 and arginase-1) in TAMs were measured by qPCR $(n=8)$. (d) Relative protein levels of M1/M2 markers in TAMs were determined by western blot $(n=8)$. (e) Production of Th1 cytokines (TNF- $\alpha$ and IL-12) and Th2 cytokines (TGF- $\beta$ and IL-10) in TAMs was determined by ELISA $(n=8)$. The value is presented as the mean \pm s.e.m. ${ }^{*} P<0.05$, ${ }^{* *} P<0.01$ vs model.

pre-metastatic niche formation and poor prognosis in patients with breast cancer. CYP4A overexpression in the TAMs promotes pre-metastatic niche formation and metastasis. Conversely, the pharmacological inhibition of CYP4A by HET0016 and DDMS decreased VEGFR1 ${ }^{+}$myeloid cell recruitment, pro-metastatic protein expression and lung metastatic burden. The focus on initiating distant organ metastasis through myeloid cells has been on tumor cell- and myeloid cell-produced factors. ${ }^{36} \mathrm{~A}$ variety of tumor-derived factors have been shown to drive the premetastatic niche formation. However, little has been done in the way of investigating the processes occurring at the primary tumor to stimulate their initial production. ${ }^{2}$ We demonstrated that CYP4A/20-HETE in TAMs regulates M2 macrophage-derived factors (TGF- $\beta$, VEGF and SDF-1), priming the microenvironment at future metastatic sites and thereby promotes metastasis. Given that CYP4A/20-HETE mediates the metastatic signals triggered by multiple of cytokines, including VEGF, SDF-1 and TGF- $\beta$, novel targeted therapies founded on CYP4A not any single cytokine may represent a potential strategy for prevention of metastasis.

Constitutive activation of STAT3 has been recognized as a crucial signal in macrophage M2 polarization. ${ }^{32}$ The inhibition of STAT3 signaling exerts anti-tumor immunity. ${ }^{37} \mathrm{~A}$ crucial role of 
a

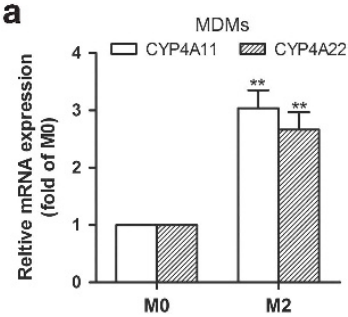

MDMs

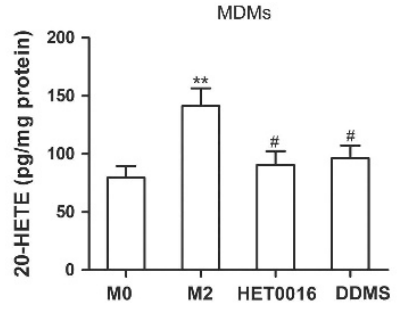

b
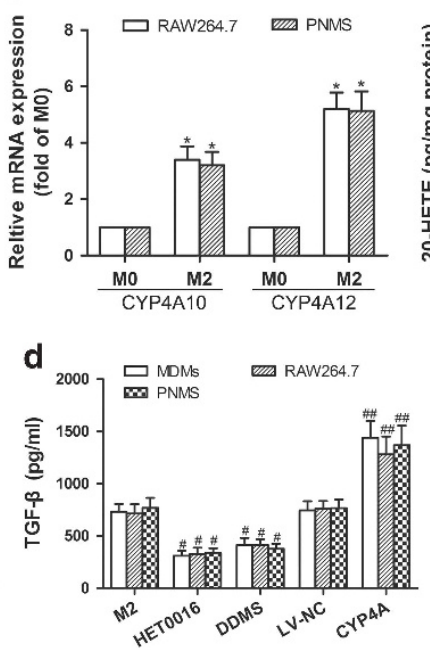
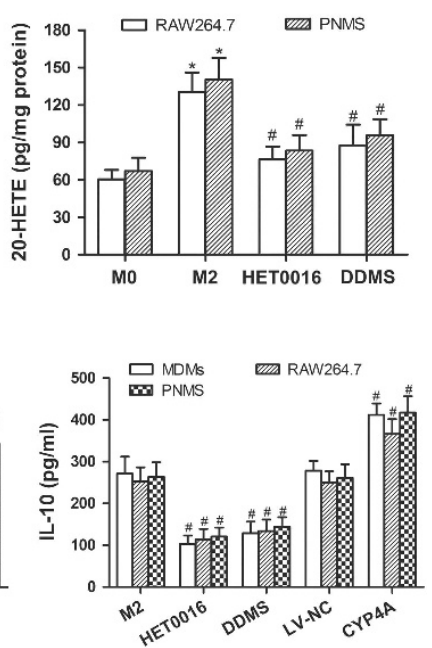

e
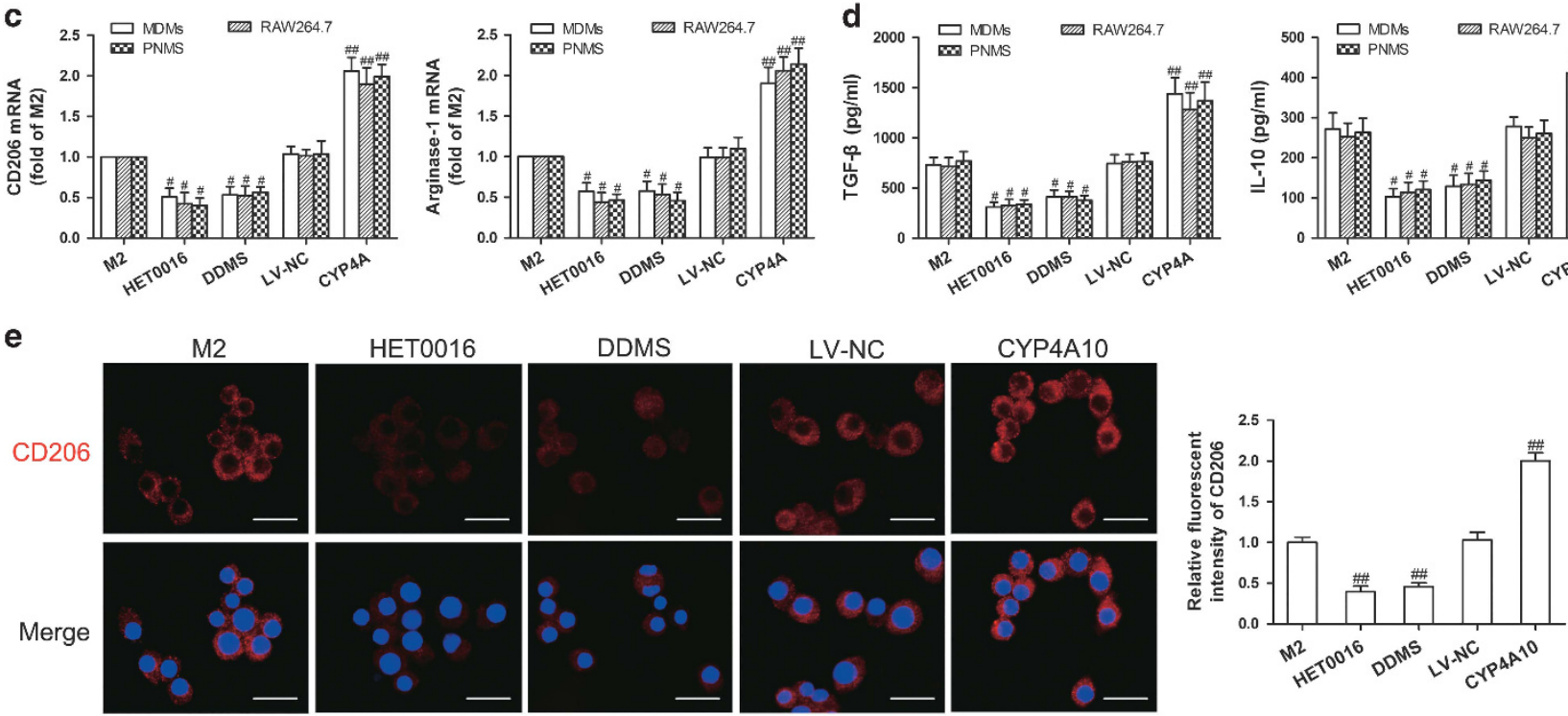

Figure 6. CYP4A contributes to macrophage M2 polarization in vitro. (a) Human monocyte-derived macrophages (MDMs) were treated with the tumor-conditioned medium (CM) for $72 \mathrm{~h}$ to generate $\mathrm{M} 2$ macrophages (M2), and then the unpolarized macrophages (M0) and $\mathrm{M} 2 \mathrm{2}$ were incubated with HET0016 $(5 \mu \mathrm{M})$, DDMS $(10 \mu \mathrm{M})$ or vehicle for $24 \mathrm{~h}$. CYP4A11/22 mRNA expression in the M0 and M2 was measured by qPCR, and 20-HETE production in M0, M2 and HET0016 or DDMS-treated M2 was determined by LC-ESI-MS/MS. (b) RAW264.7 cells and mouse peritoneal macrophages (PNMS) were treated with IL-4/IL-13 $(20 \mathrm{ng} / \mathrm{ml})$ for $12 \mathrm{~h}$ to generate $\mathrm{M} 2$, and then the $\mathrm{M0}$ and $\mathrm{M} 2 \mathrm{were}$ incubated with HET0016 (5 $\mu \mathrm{m})$, DDMS $(10 \mu \mathrm{m})$ or vehicle for $24 \mathrm{~h}$. CYP4A10/12 mRNA expression in the M0 and M2 was measured by qPCR, and 20 -HETE production in M0, M2 and HET0016 or DDMS-treated M2 was determined by LC-ESI-MS/MS. (c) Parental and CYP4A10/11 high macrophages treated with IL-4/IL-13 $(20 \mathrm{ng} / \mathrm{ml})$ for $12 \mathrm{~h}$ or the tumor CM for $72 \mathrm{~h}$ were incubated with HET0016 $(5 \mu \mathrm{M})$, DDMS (10 $\mu \mathrm{m})$ or vehicle for $24 \mathrm{~h}$. M2 markers (CD206 and arginase-1) were determined by qPCR. (d) Production of Th2 cytokines (TGF- $\beta$ and IL-10) was determined by ELISA. (e) Immunostaining analysis of CD206 (red) in PNMS from the above treated groups was shown. Scale bars, $20 \mu \mathrm{m}$. Each value presents the mean \pm s.e.m. of three independent triplicate experiments. ${ }^{*} P<0.05,{ }^{*} P<0.01$ vs $\mathrm{M} 0,{ }^{\#} P<0.05,{ }^{\# \#} P<0.01$ vs $\mathrm{M} 2$.

STAT3 in tumor metastasis has been well documented, and recent studies further indicated the importance of STAT3 signaling in formatting pre-metastatic niches in future metastatic sites. ${ }^{38}$ We demonstrated that CYP4A/20-HETE-STAT3 signaling was enhanced during the polarization of macrophages to an $M 2$ phenotype. Conversely, CYP4A inhibition decreased p-STAT3 levels, accompanied with a decrease in $M 2$ marker expression and $\mathrm{VEGFR}^{+}$myeloid cell migration. Importantly, siRNA and pharmacological inhibition of STAT3 attenuated the enhancing effects of CYP4A /20-HETE on macrophage M2 polarization and VEGFR $1^{+}$myeloid cell migration. These data suggest that CYP4A/ 20-HETE-STAT3 signaling in TAMs is crucial for lung pre-metastatic niche formation and metastasis.

Taken together, CYP4A/20-HETE in TAMs promotes premetastatic niche formation and metastasis through M2 macrophage-derived factors TGF- $\beta$, SDF- 1 and VEGF via STAT3 signaling (Figure 10). Importantly, our study identifies a previously unknown crosstalk of TAMs with VEGFR ${ }^{+}$myeloid cells regulated by CYP4A/20-HETE-STAT3 axis. Our findings suggest that CYP4A in TAMs plays a key role in pre-metastatic niche formation and metastasis, and may serve as a potential target for prevention and treatment of metastasis: target pre-metastatic niches before clinical detection of metastasis.

\section{MATERIALS AND METHODS}

Human tissue microarrays

Tissue microarrays for breast carcinoma (BRC1021) and melanoma (MEL961) were from Fanpu Biochip Company (Guilin, China). Another independent breast cancer tissue microarray (HBreD140Su03, Outdo Biotech Co., Shanghai, China) contained 140 patients in which overall survival data and RFS data were available. Each sample dot with a diameter of $1.5 \mathrm{~mm}$ and a thickness of $4 \mu \mathrm{m}$ was prepared according to a standard method. The histological diagnosis for each sample was reconfirmed using microscopic examination of hematoxylin/eosin-stained sections. Informed consent was obtained for all patients.

Patients and tissue samples

The breast tumor and matched uninvolved lymph nodes samples were acquired from 30 consenting breast cancer patients. Written informed consent was obtained from all patients, and the Institutional Review Board of the Wuhan University approved the study. None of the patients had received chemotherapy or radiotherapy at the time when tissue samples were collected. Tumors were histologically classified as invasive breast cancer according to World Health Organization (WHO) criteria.

\section{TCGA data}

We used independent cohort from TCGA, which was a publically available, open-access and clinical data (update to 24 February 2015). 
a
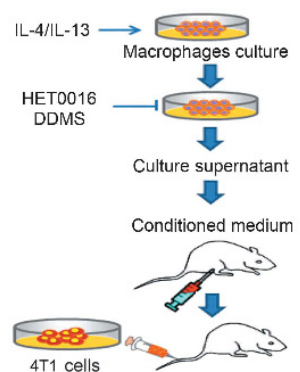

b

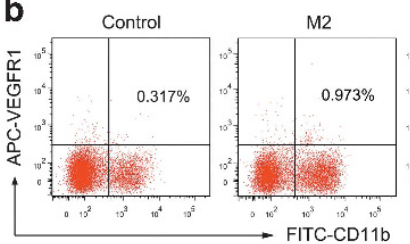

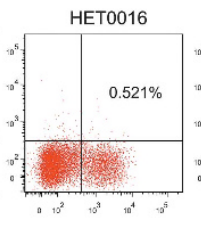

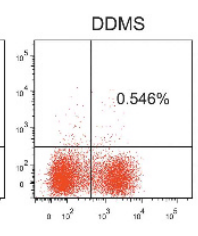

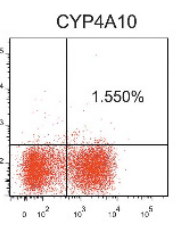

e

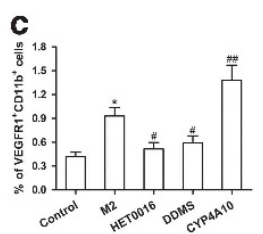

d
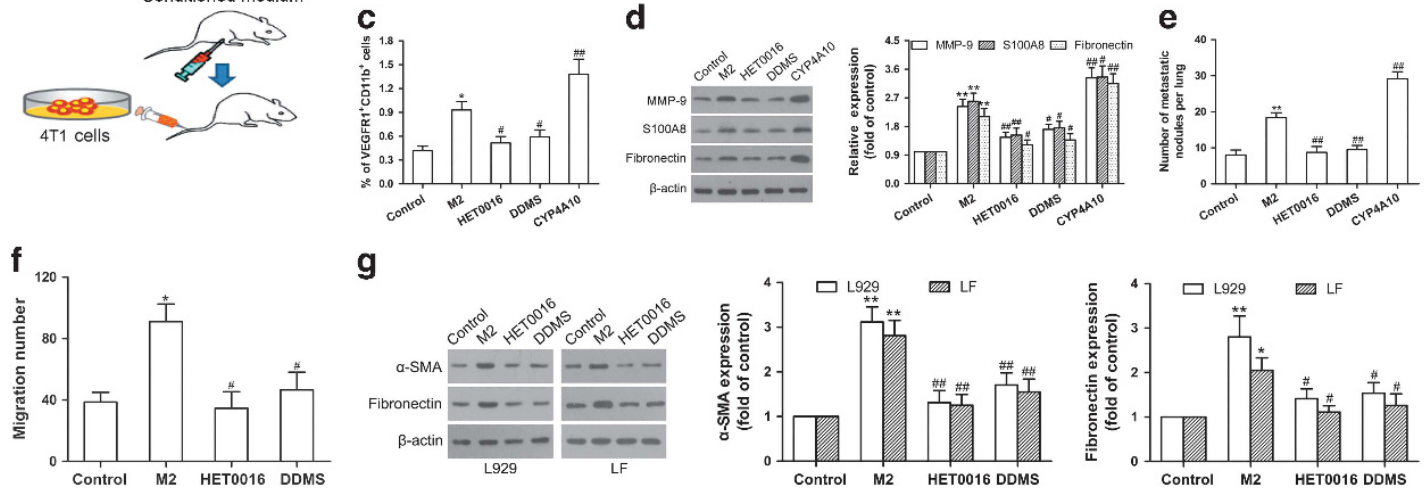

g
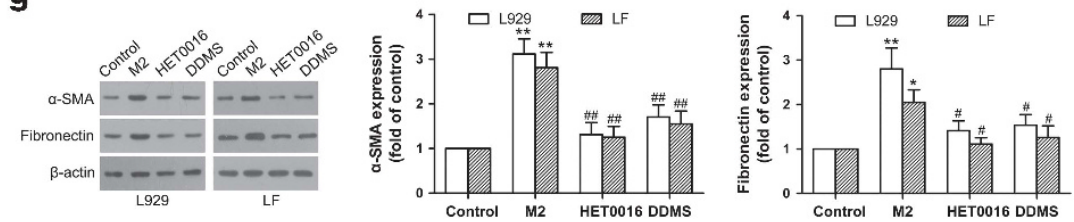

Figure 7. CYP4A promotes M2 macrophage-induced pre-metastatic niche formation and metastasis. (a) Schematic representation of the experimental approach. (b, c) BALB/c mice were intraperitoneally injected with the conditioned medium (CM) from M2 macrophages (M2), HET0016 or DDMS-treated M2, and CYP4A $10^{\text {high }}$ M2 daily for 2 weeks. Serum-free medium as control. VEGFR $1^{+}$myeloid cells in the lungs were analyzed by flow cytometry $(n=5)$. (d) MMP-9, S100A8 and fibronectin in the lungs were determined by western blot $(n=8)$. (e) In another experiment, the mice treated as described above were followed by tail vein injection of $4 \mathrm{~T} 1$ cells $\left(2 \times 10^{5}\right)$. The number of lung metastatic nodules was counted on day 10 after tumor implantation $(n=8)$. (f) RAW264.7 cells were treated with IL-4/IL-13 for $12 \mathrm{~h}$, followed by incubation with or without HET0016 $(5 \mu \mathrm{M})$ or DDMS $(10 \mu \mathrm{M})$ for another $24 \mathrm{~h}$. The CM prepared from the culture supernatants was added to the lower chamber. VEGFR $1^{+}$myeloid cells were added to the upper chamber, and then incubated over night. The cells migrated to the lower chamber were counted. (g) L929 fibroblast (L929) and lung-derived fibroblast (LF) were stimulated with above CM for 48 h. Serum-free medium as control. $\alpha$-SMA and fibronectin were measured by western blot. The value is presented as the mean \pm s.e.m. ${ }^{\#} P<0.05$, ${ }^{\# \#} P<0.01$ vs $\mathrm{M} 2,{ }^{*} P<0.05,{ }^{* *} P<0.01$ vs control.
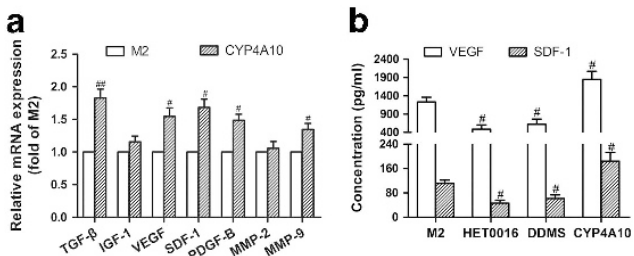

e

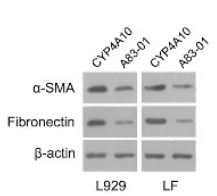

C

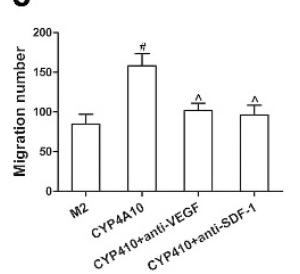

f

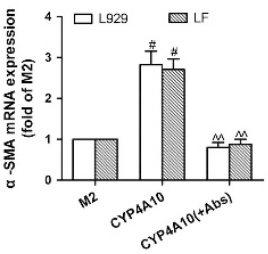

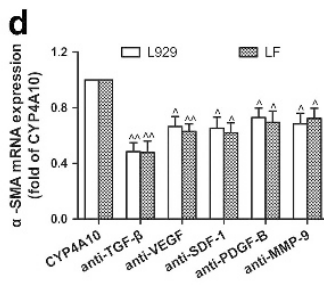

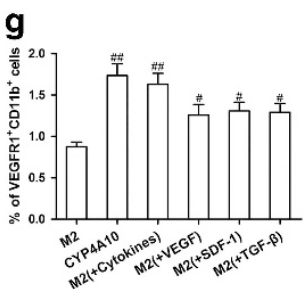

Figure 8. M2 macrophage-derived factors regulated by CYP4A drive lung pre-metastatic niche formation. (a) TGF- $\beta$, IGF-1, VEGF, SDF-1, PDGFB, MMP-2 and MMP-9 in M2 macrophages (M2) and CYP4A10 high M2 were determined by qPCR. (b) VEGF and SDF-1 from the culture supernatants were determined by ELISA. (c) The conditioned medium (CM) from M2 or CYP4A10 high M2 incubated with or without neutralizing anti-VEGF $(20 \mu \mathrm{g} / \mathrm{ml})$ or anti-SDF-1 $(10 \mu \mathrm{g} / \mathrm{ml})$ antibodies for $48 \mathrm{~h}$ was added to the lower chamber. VEGFR1 ${ }^{+}$myeloid cells were added to the upper chamber, and then incubated over night. The cells migrated to the lower chamber were counted. (d) L929 fibroblast (L929) and lung-derived fibroblast (LF) were stimulated with the CM from CYP4A $10^{\text {high }} \mathrm{M} 2$ with or without neutralizing anti-TGF- $\beta$ (10 $\left.\mu \mathrm{g} / \mathrm{ml}\right)$, anti-VEGF $(20 \mu \mathrm{g} / \mathrm{ml})$, anti-SDF-1 $(10 \mu \mathrm{g} / \mathrm{ml})$, anti-PDGF-B $(10 \mu \mathrm{g} / \mathrm{ml})$ or anti-MMP-9 $(20 \mu \mathrm{g} / \mathrm{ml})$ antibodies for $48 \mathrm{~h}$. $\alpha$-SMA was determined by GPCR. (e) L929 and LF were pre-treated with or without TGF- $\beta$ receptor inhibitor (A83-01, $10 \mu \mathrm{m})$ for $1 \mathrm{~h}$, and then incubated with the CM from CYP4A $10^{\text {high }}$ M2 for another 48 h. $\alpha$-SMA and fibronectin were measured by western blot. (f) L929 and LF were stimulated with the CM from M2 or CYP4A $10^{\text {high }}$ M2 with or without a combination of neutralizing anti-TGF- $\beta(10 \mu \mathrm{g} / \mathrm{ml})$, anti-VEGF $(20 \mu \mathrm{g} / \mathrm{ml})$ and anti-SDF- $1(10 \mu \mathrm{g} / \mathrm{ml})$ antibodies for $48 \mathrm{~h}$. $\alpha$-SMA was determined by qPCR. (g) M2 CM, CYP4A10 ${ }^{\text {high }}$ M2 CM, M2 CM plus VEGF (800 pg/ml) or SDF-1 (60 pg/ml) or TGF- $\beta(200 \mathrm{pg} / \mathrm{ml})$, and M2 CM plus combination cytokines (VEGF, SDF-1 and TGF- $\beta$ ) were intraperitoneally injected into BALB/c mice daily for 2 weeks. VEGFR $1^{+}$myeloid cells in the lungs were analyzed by flow cytometry $(n=5)$. The value is presented as the mean \pm s.e.m. ${ }^{\#} P<0.05$, ${ }^{\# \#} P<0.01$ vs M2, ${ }^{\wedge} P<0.05,{ }^{\wedge} P<0.01$ vs CYP4A10. 
a

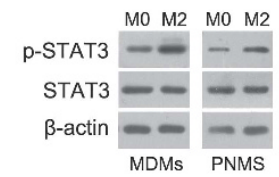

b
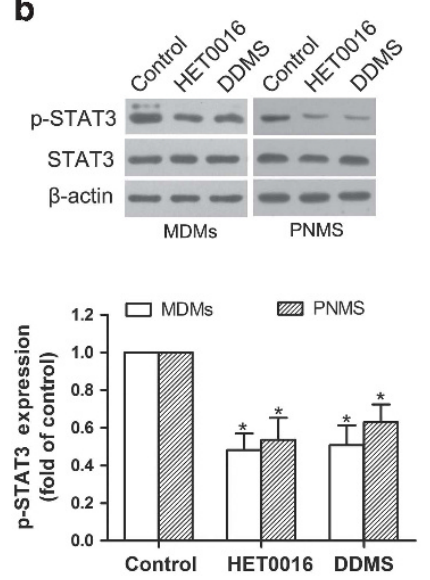

C
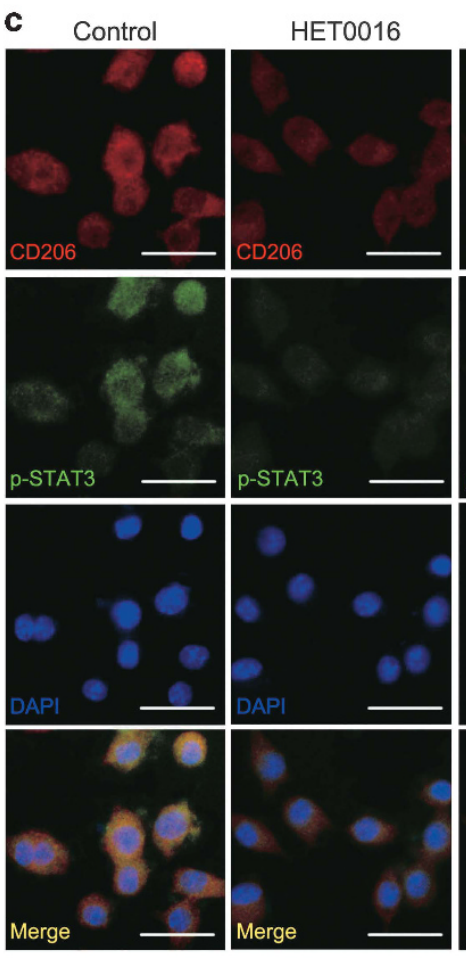
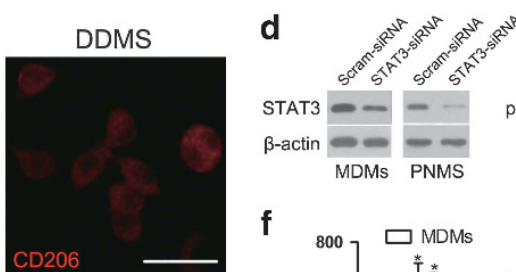

f
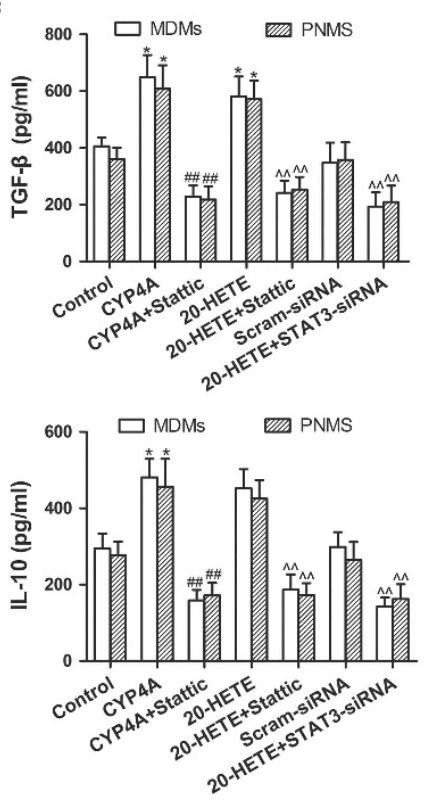
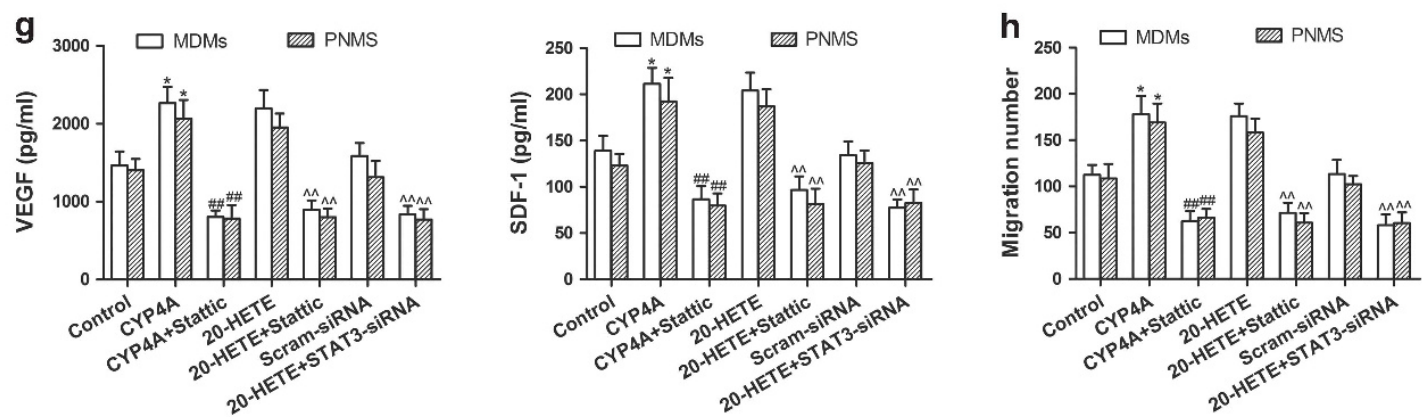

Figure 9. CYP4A/20-HETE-STAT3 signaling is crucial for M2 polarization of macrophages and migration of VEGFR $1^{+}$myeloid cells in vitro. (a, b) Mouse peritoneal macrophages (PNMS) or human monocyte-derived macrophages (MDMs) were stimulated with IL-4/IL-13 (20 ng/ml) for $12 \mathrm{~h}$ or the tumor-conditioned medium (CM) for $72 \mathrm{~h}$ to generate M2 macrophages, and then treated with HET0016 (5 $\mu \mathrm{M})$, DDMS (10 $\mu \mathrm{M})$ or vehicle for $24 \mathrm{~h}$. STAT3 and p-STAT3 was determined by western blot. (c) Immunostaining analysis of CD206 (red) and p-STAT3 (green) in PNMS from the above treated groups was shown. Scale bars, $20 \mu \mathrm{m}$. (d) The effect of siRNA against STAT3 on the protein levels of STAT3 was assayed by western blot. (e) The effect of Stattic on p-STAT3 was assayed by western blot. (f) MDMs and PNMS were pre-treated with or without STAT3 inhibitor (Stattic, $20 \mu \mathrm{M}$ ) for $1 \mathrm{~h}$, and then incubated with IL-4/IL-13 for $12 \mathrm{~h}$ or the tumor CM for $72 \mathrm{~h}$, followed by treatment with or without 20-HETE (1 $\mu \mathrm{M}$ ) for another $24 \mathrm{~h}$. In another experiment, MDMs and PNMS were pre-treated with or without STAT3 siRNA (STAT3-siRNA) and scramble siRNA for $24 \mathrm{~h}$, and then incubated with IL-4/IL-13 for $12 \mathrm{~h}$ or the tumor CM for $72 \mathrm{~h}$, followed by treatment with $20-\mathrm{HETE}(1 \mu \mathrm{M})$ for another $24 \mathrm{~h}$. Th2 cytokines (TGF- $\beta$ and IL-10) were determined by ELISA. (g) VEGF and SDF-1 from the culture supernatants in the above groups were determined by ELISA. (h) The CM in the above treated groups was added to the lower chamber. VEGFR $1^{+}$myeloid cells were added to the upper chamber, and then incubated over night. The cells migrated to the lower chamber were counted. Each value presents the mean \pm s.e.m. of three independent triplicate experiments. ${ }^{*} P<0.05,{ }^{* *} P<0.01$ vs control, ${ }^{\#} P<0.05$, \#\#P<0.01 vs CYP4A, ${ }^{\wedge} P<0.05$, ${ }^{\wedge} P<0.01$ vs 20 -HETE.

The data sets analyzed were the TCGA invasive breast carcinoma and cutaneous melanoma gene expression by RNAseq (IlluminaHiSeq, Illumina, San Diego, CA, USA). The invasive breast carcinoma cohort contained 780 patients in which overall survival data and RFS data were available. The cutaneous melanoma cohort contained 71 patients in which overall survival data and RFS data were available. Data from the TCGA were analyzed using the UCSC Cancer Genome Browser (http://genome-cancer. soe.ucsc.edu/). Invasive breast cancer and melanoma patients were grouped by CYP4A11/22 gene levels in primary tumor tissues: the $50 \%$ of patients top ranked for CYP4A11/22 levels were placed in the CYP4A11/22-high group and the bottom ranked $50 \%$ of patients in the CYP4A11/22-low group.
Chemicals and reagents

HET0016, DDMS and 20-HETE were purchased from Cayman Chemicals (Ann Arbor, MI, USA). Human recombinant macrophage colony-stimulating factor (M-CSF), mouse recombinant IL-4 and IL-13 were purchased from Pepro Tech (Rocky Hill, NJ, USA). Mouse recombinant VEGF, SDF-1 and TGF$\beta$ were purchased from R\&D Systems (Oxford, UK). Stattic (a STAT3 inhibitor) was purchased from Merck (Darmstadt, Germany). Clodronate (Clod) was purchased from Roche Diagnostics (Indianapolis, IN, USA). Zoledronic acid (ZA) and A83-01 (a TGF- $\beta$ receptor inhibitor) were purchased from Sigma Chemical Co. (St Louis, MO, USA). Neutralizing antibodies against TGF- $\beta$ (MAB7346), VEGF (AF-493) and SDF-1 (MAB310) were purchased from R\&D Systems. Neutralizing antibody against PDGF-B 


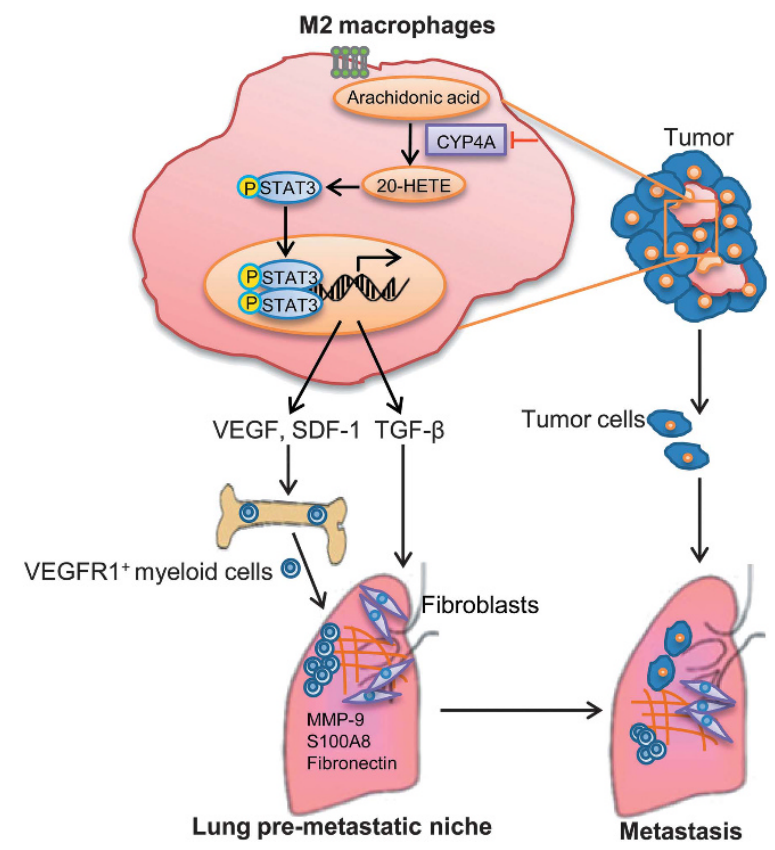

Figure 10. A proposed mechanism to explain the role of CYP4A/20HETE in TAMs in pre-metastatic niche formation and metastasis. 20-HETE, 20-hydroxyeicosatetraenoic acid; CYP4A, cytochrome P450 4A; MMP-9. matrix metalloproteinase-9; SDF-1, stromal cellderived factor-1; STAT3, signal transducer and activator of transcription 3; TAMs, tumor-associated macrophages; TGF- $\beta$, transforming growth factor- $\beta$; VEGF, vascular endothelial growth factor.

(ab34074), mCherry (ab125096) antibody and CD68 (ab955) antibody were purchased from Abcam (Cambridge, MA, USA). Neutralizing antibody against MMP-9 (MA5-13595) was purchased from Thermo Fisher Scientific (Waltham, MA, USA). F4/80 antibody (sc-377009) was purchased from Santa Cruz Biotechnology (Santa Cruz, CA, USA).

\section{Xenograft tumor models and treatment regimes}

All animal experiments were performed in accordance with the policies of the animal ethics committee of the Animal Research Committee of Wuhan University, and maintained in accordance with the guidelines by the Association for Assessment and Accreditation of Laboratory Animal Care International. BALB/c mice (female, 6-8 weeks old) and C57BL/6 mice (female, 6-8 weeks old) were purchased from Center for Disease Control and Prevention (Hubei, China), and GFP transgenic C57BL/6 mice were purchased from Shanghai Biomodel Organism Science \& Technology Development Co., Ltd (Shanghai, China). All groups were randomly divided.

For tumor inoculations, $4 \mathrm{~T} 1$-mCherry-luciferase cells $\left(1 \times 10^{6}\right)$ or B16F10mCherry-luciferase cells $\left(2 \times 10^{5}\right)$ were injected to the right mammary fat pad of BALB/c mice or the armpit of C57BL/6 mice, respectively. HET0016 $(5.0 \mathrm{mg} / \mathrm{kg})$, DDMS $(7.5 \mathrm{mg} / \mathrm{kg})$ or vehicle (DMSO) were administrated intraperitoneally five times a week, starting on the day after tumor implantation. After the mice were killed on day 14 or day 28 peripheral blood, tumors and lungs were collected and analyzed. Lung metastasis was measured by hematoxylin/eosin and mCherry staining. Pulmonary metastatic nodules were counted in three serial sections. Lung metastasis also detected by ex vivo luciferase based noninvasive bioluminescence imaging system (In-Vivo Xtreme II, Bruker, Billerica, MA, USA). The lung images were analyzed by quantification of total photon flux of each lung using Molecular Imaging Software (Bruker).

In another experiment, bone marrow-derived cells were harvested from GFP transgenic C57BL/ 6 mice, and then the VEGFR1 ${ }^{+} \mathrm{GFP}^{+}$myeloid cells were isolated from bone marrow-derived cells by flow cytometry with purities of $>95 \%$. The B16F10-bearing mice treated with or without HET0016 $(5.0 \mathrm{mg} / \mathrm{kg})$ or DDMS $(7.5 \mathrm{mg} / \mathrm{kg})$ were injected with VEGFR $1^{+} \mathrm{GFP}^{+}$myeloid cells by tail vein on day 12 after tumor implantation. After $48 \mathrm{~h}, \mathrm{VEGFR1}{ }^{+} \mathrm{GFP}^{+}$myeloid cells in the lungs were analyzed by flow cytometry.
To confirm that any beneficial effects observed reflect TAM depletion rather than off-target effects of the drug, we used two complementary methods to deplete macrophage. The Clod $(100 \mathrm{mg} / \mathrm{kg})$ liposomes were administrated intraperitoneally $24 \mathrm{~h}$ after $4 \mathrm{~T} 1$ cells $\left(1 \times 10^{6}\right)$ were injected to the mammary fat pad of BALB/c mice, followed by repeated injections of $50 \mathrm{mg} / \mathrm{kg}$ every fourth day. In another experiment, ZA $(100 \mu \mathrm{g} / \mathrm{kg})$ liposomes were injected intravenously once a week, and then HET0016 $(5.0 \mathrm{mg} / \mathrm{kg})$ was administrated intraperitoneally five times a week. Clod and ZA doses were based on the literature ${ }^{39,40}$ and the efficiency of macrophage depletion was assessed by flow cytometry analysis of CD $11 \mathrm{~b}^{+}$ cells in peripheral blood and immunohistochemical analysis of tumor sections for $\mathrm{F} 4 / 80$.

In order to investigate the effects of macrophage $M 2$ polarization regulated by CYP4A on pre-metastatic niche formation and metastasis, the mice were treated by intraperitoneal injection of the CM $(300 \mu \mathrm{l})$ from control, M2 macrophages (M2) treated with or without HET0016 (5 $\mu \mathrm{M})$, DDMS $(10 \mu \mathrm{M})$ or CYP4A10 high $\mathrm{M} 2$ daily for 2 weeks, followed by tail vein injection of $4 \mathrm{~T} 1$ cells $\left(2 \times 10^{5}\right)$. The lungs were harvested and analyzed by flow cytometry on day 14 . Lung metastasis was measured by hematoxylin/ eosin staining on day 24.

For the in vivo co-injection model, $6 \times 10^{5} 4 \mathrm{~T} 1$ cells were mixed with $2 \times 10^{5}$ wild-type RAW264.7 cells, negative control lentivirus (LV-NC) RAW264.7 cells or CYP4A10 high RAW264.7 cells at a ratio of 3:1 and orthotopically injected into BALB/c mice. On day 14 or day 28 , tumors and lungs were collected and analyzed.

Cell culture, lentiviral transduction, preparation of $\mathrm{CM}$, cell proliferation assay, migration assay, flow cytometry, gene expression analysis, immunoblotting assay, measurement of 20-HETE, immunohistochemisty and tissue microarray analysis, immunofluorescence, enzyme-linked immunosorbent assay and statistical analysis were described in Supplementary Materials and Methods.

\section{CONFLICT OF INTEREST}

The authors declare no conflict of interest.

\section{ACKNOWLEDGEMENTS}

This work was supported by the National Natural Science Foundation of China (grant nos 30973552, 81272464, 81173089 (to JY), 81402958 (to WY) and 81201261 (to JZ)), the Fundamental Research Funds for the Central Universities (grant no. 2014301020201) (to XC) and Hubei Key Laboratory of Medical Information Analysis \& Tumor Diagnosis and Treatment (PJS140011508) (to CW).

\section{REFERENCES}

1 Gupta GP, Massagué J. Cancer metastasis: building a framework. Cell 2006; 127: 679-695.

2 Sceneay J, Smyth MJ, Möller A. The pre-metastatic niche: finding common ground. Cancer Metastasis Rev 2013; 32: 449-464.

3 Liu Y, Cao X. Characteristics and significance of the pre-metastatic niche. Cancer Cell 2016; 30: 668-681.

4 Kaplan RN, Riba RD, Zacharoulis S, Bramley AH, Vincent L, Costa C et al. VEGFR1-positive haematopoietic bone marrow progenitors initiate the premetastatic niche. Nature 2005; 438: 820-827.

5 Joyce JA, Pollard JW. Microenvironmental regulation of metastasis. Nat Rev Cancer 2009; 9: 239-252.

6 Chen P, Bonaldo P. Role of macrophage polarization in tumor angiogenesis and vessel normalization: implications for new anticancer therapies. Int Rev Cell Mol Biol 2013; 301: 1-35.

7 Kitamura T, Qian B-Z, Pollard JW. Immune cell promotion of metastasis. Nat Rev Immunol 2015; 15: 73-86.

8 Jia X, Yu F, Wang J, Iwanowycz S, Saaoud F, Wang Y et al. Emodin suppresses pulmonary metastasis of breast cancer accompanied with decreased macrophage recruitment and M2 polarization in the lungs. Breast Cancer Res Treat 2014; 148 : 291-302.

9 Ding L, Liang G, Yao Z, Zhang J, Liu R, Chen $\mathrm{H}$ et al. Metformin prevents cancer metastasis by inhibiting M2-like polarization of tumor associated macrophages. Oncotarget 2015; 6: 36441-36455.

10 Lewis CE, Pollard JW. Distinct role of macrophages in different tumor microenvironments. Cancer Res 2006; 66: 605-612.

11 Wculek SK, Malanchi I. Neutrophils support lung colonization of metastasisinitiating breast cancer cells. Nature 2015; 528: 413-417. 
12 Gil-Bernabé AM, Ferjančič Š, Tlalka M, Zhao L, Allen PD, Im JH et al. Recruitment of monocytes/macrophages by tissue factor-mediated coagulation is essential for metastatic cell survival and premetastatic niche establishment in mice. Blood 2012; 119: 3164-3175.

13 Sharma SK, Chintala NK, Vadrevu SK, Patel J, Karbowniczek M, Markiewski MM. Pulmonary alveolar macrophages contribute to the premetastatic niche by suppressing antitumor T cell responses in the lungs. J Immunol 2015; 194: 5529-5538.

14 Alexanian A, Sorokin A. Targeting 20-HETE producing enzymes in cancer-rationale, pharmacology, and clinical potential. Onco Targets Ther 2013; 6: 243.

15 Alexanian A, Miller B, Roman RJ, Sorokin A. 20-HETE-producing enzymes are up-regulated in human cancers. Cancer Genomics Proteomics 2012; 9: 163-169.

16 Edson KZ, Rettie AE. CYP4 enzymes as potential drug targets: focus on enzyme multiplicity, inducers and inhibitors, and therapeutic modulation of 20-hydroxyeicosatetraenoic acid (20-HETE) synthase and fatty acid $\omega$-hydroxylase activities. Curr Topics Med Chem 2013; 13: 1429-1440.

17 Borin TF, Zuccari DA, Jardim-Perassi BV, Ferreira LC, Iskander A, Varma NRS et al. HET0016, a selective inhibitor of 20-HETE synthesis, decreases pro-angiogenic factors and inhibits growth of triple negative breast cancer in mice. PLoS One 2014; 9: e116247.

18 Yu W, Chen L, Yang Y-Q, Falck JR, Guo AM, Li Y et al. Cytochrome P450 $\omega$-hydroxylase promotes angiogenesis and metastasis by upregulation of VEGF and MMP-9 in non-small cell lung cancer. Cancer Chemother Pharmacol 2011; 68: 619-629.

19 Zheng H, Li Y, Wang Y, Zhao H, Zhang J, Chai H et al. Downregulation of COX-2 and CYP 4A signaling by isoliquiritigenin inhibits human breast cancer metastasis through preventing anoikis resistance, migration and invasion. Toxicol Appl Pharmacol 2014; 280: 10-20.

20 Kroetz DL, Xu F. Regulation and inhibition of arachidonic acid $\omega$-hydroxylases and 20-HETE formation. Annu Rev Pharmacol Toxicol 2005; 45: 413-438.

21 Zhao H, Zhang X, Chen X, Li Y, Ke Z, Tang T et al. Isoliquiritigenin, a flavonoid from licorice, blocks M2 macrophage polarization in colitis-associated tumorigenesis through downregulating $\mathrm{PGE}_{2}$ and IL-6. Toxicol Appl Pharmacol 2014; 279: 311-321.

22 Giles AJ, Reid CM, Evans JD, Murgai M, Vicioso Y, Highfill SL et al. Activation of hematopoietic stem/progenitor cells promotes immunosuppression within the pre-metastatic niche. Cancer Res 2016; 76: 1335-1347.

23 Pal SK, Vuong W, Zhang W, Deng J, Liu X, Carmichael C et al. Clinical and translational assessment of VEGFR1 as a mediator of the premetastatic niche in high-risk localized prostate cancer. Mol Cancer Ther 2015; 14: 2896-2900.

24 Hiratsuka S, Watanabe A, Aburatani H, Maru Y. Tumour-mediated upregulation of chemoattractants and recruitment of myeloid cells predetermines lung metastasis. Nat Cell Biol 2006; 8: 1369-1375.

25 Acuff HB, Carter KJ, Fingleton B, Gorden DL, Matrisian LM. Matrix metalloproteinase-9 from bone marrow-derived cells contributes to survival but not growth of tumor cells in the lung microenvironment. Cancer Res 2006; 66: 259-266.

26 Wu CF, Andzinski L, Kasnitz N, Kröger A, Klawonn F, Lienenklaus S et al. The lack of type I interferon induces neutrophil-mediated pre-metastatic niche formation in the mouse lung. Int J Cancer 2015; 137: 837-847.

27 Fang W, Ye L, Shen L, Cai J, Huang F, Wei Q et al. Tumor-associated macrophages promote the metastatic potential of thyroid papillary cancer by releasing CXCL8. Carcinogenesis 2014; 35: 1780-1787.
28 Chen Y, Gou X, Kong DK, Wang X, Wang J, Chen Z et al. EMMPRIN regulates tumor growth and metastasis by recruiting bone marrow-derived cells through paracrine signaling of SDF-1 and VEGF. Oncotarget 2015; 6: 32575-32585.

29 Cirri P, Chiarugi P. Cancer associated fibroblasts: the dark side of the coin Am J Cancer Res 2011; 1: 482-497.

30 Sun Q, Liu L, Mandal J, Molino A, Stolz D, Tamm M et al. PDGF-BB induces PRMT1 expression through ERK1/2 dependent STAT1 activation and regulates remodeling in primary human lung fibroblasts. Cell Signal 2016; 28: 307-315.

31 Dayer C, Stamenkovic I. Recruitment of matrix metalloproteinase-9 (MMP-9) to the fibroblast cell surface by lysyl hydroxylase 3 (LH3) triggers transforming growth factor- $\beta$ (TGF- $\beta$ ) activation and fibroblast differentiation. J Biol Chem 2015; 290: 13763-13778.

32 Wei J, Besner GE. M1 to M2 macrophage polarization in heparin-binding epidermal growth factor-like growth factor therapy for necrotizing enterocolitis. J Surg Res 2015; 197: 126-138.

33 Costa-Silva B, Aiello NM, Ocean AJ, Singh S, Zhang H, Thakur BK et al. Pancreatic cancer exosomes initiate pre-metastatic niche formation in the liver. Nat Cell Biol 2015; 17: 816-826.

34 Liu Y, Gu Y, Han Y, Zhang Q, Jiang Z, Zhang X et al. Tumor exosomal RNAs promote lung pre-metastatic niche formation by activating alveolar epithelial TLR3 to recruit neutrophils. Cancer Cell 2016; 30: 243-256.

35 Kowanetz M, Wu X, Lee J, Tan M, Hagenbeek T, Qu X et al. Granulocyte-colony stimulating factor promotes lung metastasis through mobilization of $\mathrm{Ly} 6 \mathrm{G}^{+} \mathrm{Ly}_{6 \mathrm{C}} \mathrm{C}^{+}$ granulocytes. Proc Natl Acad Sci USA 2010; 107: 21248-21255.

36 Kitamura T, Qian B-Z, Pollard JW. Immune cell promotion of metastasis. Nat Rev Immunol 2015; 15: 73-86.

37 Kortylewski M, Kujawski M, Wang T, Wei S, Zhang S, Pilon-Thomas S et al. Inhibiting Stat3 signaling in the hematopoietic system elicits multicomponent antitumor immunity. Nat Med 2005; 11: 1314-1321.

38 Deng J, Liu Y, Lee H, Herrmann A, Zhang W, Zhang C et al. S1PR1-STAT3 signaling is crucial for myeloid cell colonization at future metastatic sites. Cancer Cell 2012 21: 642-654.

39 Rolny C, Mazzone M, Tugues S, Laoui D, Johansson I, Coulon C et al. HRG inhibits tumor growth and metastasis by inducing macrophage polarization and vessel normalization through downregulation of PIGF. Cancer Cell 2011; 19: 31-44.

40 Zhang W, Zhu X-D, Sun H-C, Xiong Y-Q, Zhuang P-Y, Xu H-X et al. Depletion of tumor-associated macrophages enhances the effect of sorafenib in metastatic liver cancer models by antimetastatic and antiangiogenic effects. Clin Cancer Res 2010; 16: 3420-3430.

(c) (i) $\Theta$ This work is licensed under a Creative Commons AttributionNonCommercial-NoDerivs 4.0 International License. The images or other third party material in this article are included in the article's Creative Commons license, unless indicated otherwise in the credit line; if the material is not included under the Creative Commons license, users will need to obtain permission from the license holder to reproduce the material. To view a copy of this license, visit http:// creativecommons.org/licenses/by-nc-nd/4.0/

(c) The Author(s) 2017

Supplementary Information accompanies this paper on the Oncogene website (http://www.nature.com/onc) 